\title{
Contribution à l'étude faunistique et écologique des simulies (Diptera : Simuliidae) du Maroc IV. Les simulies du Haut Atlas. Description d'une nouvelle espèce
}

\author{
J. Giudicelli ${ }^{1}$ \\ A. Bouzidi ${ }^{2}$ \\ N. Ait Abdelaali ${ }^{3}$
}

Mots clés : Diptera, Simuliidae, Haut Atlas, Maroc, espèce nouvelle, eaux courantes, biogéographie, écologie, zonation.

Des simulies ont été récoltées entre 1985 et 1991 dans les principaux réseaux hydrographiques du Haut Atlas. Les stades préimaginaux des simulies représentent un élément important de la faune benthique de ces cours d'eau. Des larves et des nymphes ont été récoltées dans 54 stations (entre $450 \mathrm{~m}$ et $3800 \mathrm{~m}$ d'altitude) qui regroupent une grande variété d'eaux courantes, depuis les ruisselets jusqu'aux grandes rivières.

La communauté des simulies du Haut Atlas compte 24 espèces ; six sont nouvelles pour la science. Cinq espèces ont été décrites précédemment. La description de Simulium (Eusimulium) mellah Giudicelli \& Bouzidi n. sp. est donnée dans le présent travail.

Le nombre d'espèces de simulies connues actuellement du Haut Atlas est remarquablement élevé, comparé à celui d'autres régions d'Europe et du bassin méditerranéen ; à l'inverse, on note une relative pauvreté en espèces dans les autres groupes d'invertébrés vivant dans les eaux courantes de ce massif.

Beaucoup d'espèces sont communes avec la faune des simulies de l'Europe occidentale et surtout de l'Europe méditerranéenne. La faune des simulies du Haut Atlas est nettement paléarctique, dominée par des éléments à distribution ouest-méditerranéenne et circumméditerranéenne ; elle comprend aussi quelques éléments africains. Quatre espèces sont endémiques, cantonnées dans les torrents et les sources de haute altitude.

Des informations sur la biogéographie, l'écologie et la répartition des espèces sont fournies.

Les affinités et les relations écologiques entre 23 espèces et 50 stations ont été déterminées par une analyse factorielle des correspondances. Cinq groupements d'espèces et de stations ont été reconnus : un premier groupement caractérise les cours d'eau froids (torrents et sources) aux altitudes supérieures (entre 2660 et $3200 \mathrm{~m}$ ) ; les deuxième et troisième groupements correspondent au crénal et aux secteurs initiaux du rhithral (entre 1600 et $2700 \mathrm{~m}$ ) et à une communauté d'espèces sténothermes d'eau froide ; le quatrième groupement réunit les espèces eurythermes à large répartition dans les réseaux hydrographiques et il correspond aux rivières de montagne (hypo-métarhithral) entre 1200 et $2600 \mathrm{~m}$ d'altitude ; le cinquième groupement contient les espèces thermophiles et potamophiles qui habitent le cours inférieur des rivières (entre 600 et $1600 \mathrm{~m}$ ).

Une analyse factorielle des correspondances a été réalisée sur des prélèvements mensuels d'invertébrés récoltés de déc. 1985 à déc. 1986 dans les différents microhabitats, définis par la combinaison courant-substrat, de deux secteurs épipotamiques. Le premier axe factoriel (F1) représente une échelle de vitesse de courant. Les stades aquatiques des 7 espèces de Simulies présentes se disposent le long de cet axe en fonction de leur rhéopréférendum.

1. Laboratoire d'Ecologie des Eaux Continentales Méditerranéennes, Faculté des Sciences de Saint-Jérôme, 13397 Marseille cedex 20 , France.

2. Département de Biologie Appliquée, Faculté des Sciences et Techniques, Université Hassan I, BP 577, Settat, Maroc.

3. Laboratoire d'Hydrobiologie, Faculté des Sciences, Université Cadi Ayyad, B.P. 515, Marrakech, Maroc. 


\section{Contribution to the faunistic and ecological study of the blackflies (Diptera: Simuliidae) of Morocco} IV. The blackflies of the High Atlas mountain range. Description of a new species

Keywords : Diptera, Simuliidae, High Atlas, Morocco, new species, running waters, biogeography, ecology, zonation.

Blackflies have been collected along the main river systems of the High Atlas range between 1985 and 1991. Immature instars of Simuliidae were important members of the benthic fauna in these streams. Larvae and pupae were collected from 54 sampling stations (between 450 and $3800 \mathrm{~m}$ a.s.l.) which included a great diversity of running waters from trickles to rather large rivers.

The blackfly community consists of 24 species ; six species are new to science. Five species were previously described. A complete description of Simulium (Eusimulium) mellah Giudicelli \& Bouzidi sp. n. is given in the present paper.

The number of blackfly species presently recorded from High Atlas is remarkably high in comparison to several European and Mediterranean countries ; conversely, a relative scarcity of species has been observed for the other faunal groups inhabiting the High Atlas running waters.

Many species are shared with the blackfly fauna of western Europe and most of Mediterranean Europe. The blackfly fauna of High Atlas clearly appears palearctic, dominated by elements of west-mediterranean and circummediterranean distribution; it also includes some african ones. Four species are endemic; they live in high-altitude torrential streams and springs.

Information on the biogeography, ecology and distribution of the species are given.

Ecological affinities and relations between the 50 stations and 23 selected species have been determined by means of a Correspondance Analysis. Five clusters of species and stations have been recognized : a first cluster characterizes the cold-water torrential streams and spring brooks at the highest altitudes ( 2660 to $3200 \mathrm{~m}$ a.s.l.); the 2 nd and 3rd clusters correspond to the crenal and the initial sectors of the rhithral (between 1600 and $2700 \mathrm{~m}$ a.s.l.) and to a community of cold-stenothermous species; the 4th cluster is a community of eurythermic widely distributed species corresponding to mountain rivers (hypo-métarhithral) between 1200 and $2600 \mathrm{~m}$ a.s.l. ; the 5th cluster includes the thermophilous and potamophilous species and their habitat which is related to the lower course of the rivers (between 600 and $1600 \mathrm{~m}$ a.s.l.).

A factorial correspondances analysis was carried out on samples of invertebrates, collected monthly from dec. 1985 to dec. 1986 , in various microhabitats (defined by current velocity and substratum) of two epipotamic river stretches. The first factorial axis (F1) represent a current velocity scale. The immature stages of 7 blackfly species are arranged on this axis in a defined order in terms of their rheopreferendum.

\section{Introduction}

Dans la plupart des écosystèmes d'eau courante, les Diptères Simuliidés constituent souvent le groupe qui domine en effectif la communauté d'invertébrés. Néanmoins, ces Diptères sont rarement pris en compte dans les études hydrobiologiques. Les difficultés de détermination de leurs stades aquatiques expliquent, en grande partie, cette situation. Il existe quelques travaux où le peuplement simulidien a été déterminé jusqu'à l'espèce et intégré, au même titre que les autres groupes taxonomiques, dans l'analyse des écosystèmes lotiques; citons Giudicelli (1968) à propos des cours d'eau de Corse, Krno (1982) dans une étude sur les cours d'eau des Tatras de Tchékosloyaquie, Vinçon (1987) dans une étude sur les peuplements de deux réseaux hydrographiques des Pyrénées.

Dans une étude hydrobiologique concernant les principaux réseaux hydrographiques du Haut Atlas nous avons pris en compte les Diptères Simuliidés, non seulement parce qu'ils ont été récoltés dans tous les cours d'eau et à toutes les altitudes, mais surtout à cause de leur diversité taxonomique élevée (24 espèces).
La richesse taxonomique du peuplement simulidien du Haut Atlas est remarquable, comparée à celle des autres groupes d'invertébrés. Elle est identique à celle des Trichoptères, supérieure à celle des Ephéméroptères et des Plécoptères. Dans les réseaux des autres régions naturelles de la zone paléarctique la faune des Simulies est comparativement moins riche et les autres groupes taxonomiques présentent une diversité bien supérieure à celle des Simulies.

Le tableau 1 montre la richesse spécifique de la faune des Simulies, comparée à celle des autres groupes d'invertébrés, dans différents cours d'eau du bassin de la Méditerranée occidentale.

On doit à Grenier (1953) le premier inventaire des Simulies d'Afrique du Nord.

Dans la Limnofauna Europaea, H.Zwick (1978) relevait, dans la zone $\mathrm{X}$ qui correspond à l'Afrique du Nord, 13 espèces dont la présence est certaine et 16 espèces dont la présence est probable.

En Tunisie, 18 espèces sont connues (Boumaiza \& Clergue-Gazeau 1986). Les Simulies d'Algérie ont fait l'objet de deux courtes notes (Edwards 1923, Parrot 1949) ; plus récemment, Gagneur \& Clergue-Gazeau 
Tableau 1. Comparaison de la richesse spécifique des Simuliidae et des principaux groupes d'invertébrés dans les cours d'eau du Haut Atlas, du Moyen Atlas et de deux vallées des Pyrénées françaises.

Table 1. Comparison of specific richness of Simuliidae and main invertebrate groups in the streams of High Atlas, Middle Atlas and two valleys of the French Pyrenees.

\begin{tabular}{lcccccc}
\hline & Ephémères & Plécoptères & Trichoptères & $\begin{array}{c}\text { Coléoptères } \\
\text { Elmidae }\end{array}$ & $\begin{array}{c}\text { Diptères } \\
\text { Simuliidae }\end{array}$ & Total \\
\hline $\begin{array}{l}\text { Vallée d'Ossau } \\
\text { (Pyrénées) }\end{array}$ & 30 & 54 & 55 & 11 & 23 & 173 \\
$\begin{array}{l}\text { Neste d'Aure } \\
\text { Pyrénées) }\end{array}$ & 26 & 56 & 111 & 14 & 31 & 238 \\
$\begin{array}{l}\text { Moyen Atlas } \\
\text { (Maroc) }\end{array}$ & 25 & 10 & 46 & 12 & 8 & 101 \\
$\begin{array}{l}\text { Haut Atlas } \\
\text { (Maroc) }\end{array}$ & 21 & 12 & 34 & 6 & 24 & 97 \\
\hline
\end{tabular}

(1988) ont recensé 20 espèces dans les cours d'eau du nord-ouest de l'Algérie.

Les récoltes de Simulies au Maroc sont restées limitées dans l'espace et dans le temps. Elles ont donné matière à des publications épisodiques consacrées surtout à des descriptions d'espèces nouvelles. Les plus. anciennes sont celles de Grenier et al. (1957), Bernard et al (1972).

Dans les travaux plus récents :

- Bailly-Choumara \& Beaucournu-Saguez (1978, 1981) ont publié les résultats de leurs prospections dans le Rif (67 stations réparties entre 0 et $2000 \mathrm{~m}$ d'altitude), puis dans le Haut Atlas (99 stations entre 0 et $3800 \mathrm{~m}$ ). Ainsi, 15 espèces ont été recensées dans le Rif et 18 dans le Haut Atlas. Elles ont apporté des nouveautés faunistiques (description de 5 espèces nouvelles) et fourni des informations sur l'écologie de certaines espèces.

- Dakki (1979) mentionnait la présence de 8 espèces dans le réseau supérieur de l'oued Sebou (Moyen Atlas)

- Giudicelli \& Dakki (1984), à la suite de récoltes dans les ruisseaux de source du Rif, signalaient pour la première fois la présence au Maroc du sous-genre Obuchovia et de Simulium lamachi.

- Beaucournu-Saguez \& Braverman (1987) ont signalé la présence au Maroc de Grenieria fabri, sans aucune précision sur la localité de récolte.

- Nos récoltes dans le Haut Atlas, de 1985 à 1991, ont permis de recenser dans les cours d'eau de ce massif 24 espèces, dont six nouvelles pour la science. Cinq de ces espèces nouvelles ont été décrites entre 1985 et
1989 ; la sixième est décrite dans le présent travail. Deux espèces sont signalées pour la première fois en Afrique du Nord.

- Le dernier bilan sur la faune des Simulies d'Afrique du Nord (Clergue-Gazeau et al. 1991), qui intègre nos résultats mentionnés dans la thèse non publiée de A. Bouzidi (1989), fait état de 29 espèces au Maroc.

Le matériel que nous avons récolté dans le Haut Atlas, constitué de larves et de pupes, provient de prélèvements de faune benthique réalisés au filet de type Surber (surface échantillonnée: $0,1 \mathrm{~m}^{2}$ ). Des imagos ont été obtenues par élevages de pupes ; elles nous ont permis de confirmer les déterminations des stades aquatiques.

\section{Inventaire des stations prospectées}

Nous avons prélevé la faune benthique dans une soixantaine de stations dont 54 hébergeaient des larves et pupes de Simulies. Ces stations sont réparties sur les principaux réseaux hydrographiques des deux versants du Haut Atlas (Fig. 1, 2). Dans le Haut Atlas, les cours d'eau s'appellent soit «oued» (nom arabe), soit «assif» (nom berbère).

\subsection{Stations situées sur le versant nord}

- Réseau hydrographique de l'oued Réghaya

-1. assif Ait Mizane, ruisseau formé par les eaux de fonte de névés; $3800 \mathrm{~m}$.

-2. l'oued Reghaya; au niveau du refuge Neltner; $3200 \mathrm{~m}$. 


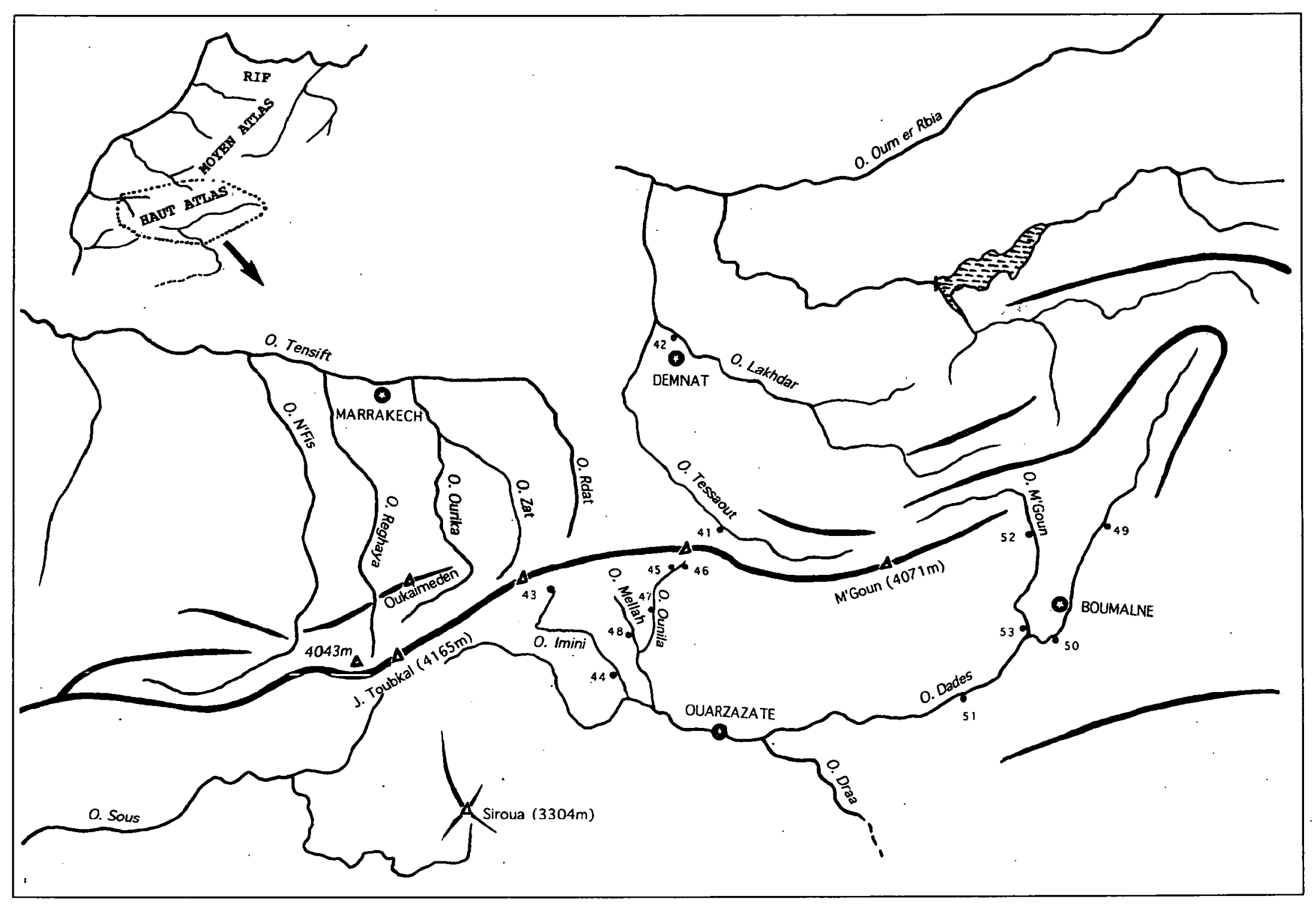

Fig. 1. Cartes des réseaux hydrographiques du Haut Atlas et localisation des stations de prélèvements des Simulies.

Fig. 1. Map showing the river systems of High Atlas and the sites at which Simuliidae were collected.

-3. l'oued Reghaya; au niveau de Sidi Chamharouch; $2300 \mathrm{~m}$.

-4. l'oued Reghaya; au niveau d'Imlil; 1740 m.

- 5. l'oued Reghaya; au niveau d'Aguersioual; $1550 \mathrm{~m}$.

-6. l'oued Reghaya; au niveau de Moulay Brahim; $1200 \mathrm{~m}$

- 7. l'oued Reghaya; en amont de Tahanaout; 890 m.

- 8. ruisselet émissaire de source débouchant dans l'oued Reghaya en amont d'Imlil; $1750 \mathrm{~m}$.

- 9. ruisselet émissaire de source débouchant dans l'assif M'zik, affluent de l'oued Reghaya; 1850 m.

\section{- Réseau de l'oued N'fis.}

- 10. ruisselet émissaire de source débouchant dans l'assif N'Ouarzane, affluent de l'oued N'fis; 3000 m.
-11. ruisseau émissaire de source formant le début de l'assif N'Ouarzane; $3000 \mathrm{~m}$.

- 12. l'assif N'Ouarzane, en aval des cascades d'Irhoulidene; $2800 \mathrm{~m}$.

-13. ruisseau affluent en rive droite de l'assif N'Ouarzane; $2400 \mathrm{~m}$.

-14. l'oued N'fis, en amont d'Ijoukak; $1600 \mathrm{~m}$.

- 15. l'oued N'fis, en amont de Wirgane; $1200 \mathrm{~m}$.

-16. l'oued N'fis; 980 m.

- 17. l'oued N'fis, en amont de la retenue de Lalla Takerkoust; $660 \mathrm{~m}$.

\section{- Réseau de l'oued Ourika.}

- 18. ruisseau de Tinzart (émissaire de source); $2850 \mathrm{~m}$.

- 19. ruisseau de Tifni (émissaire de source); $2780 \mathrm{~m}$. 

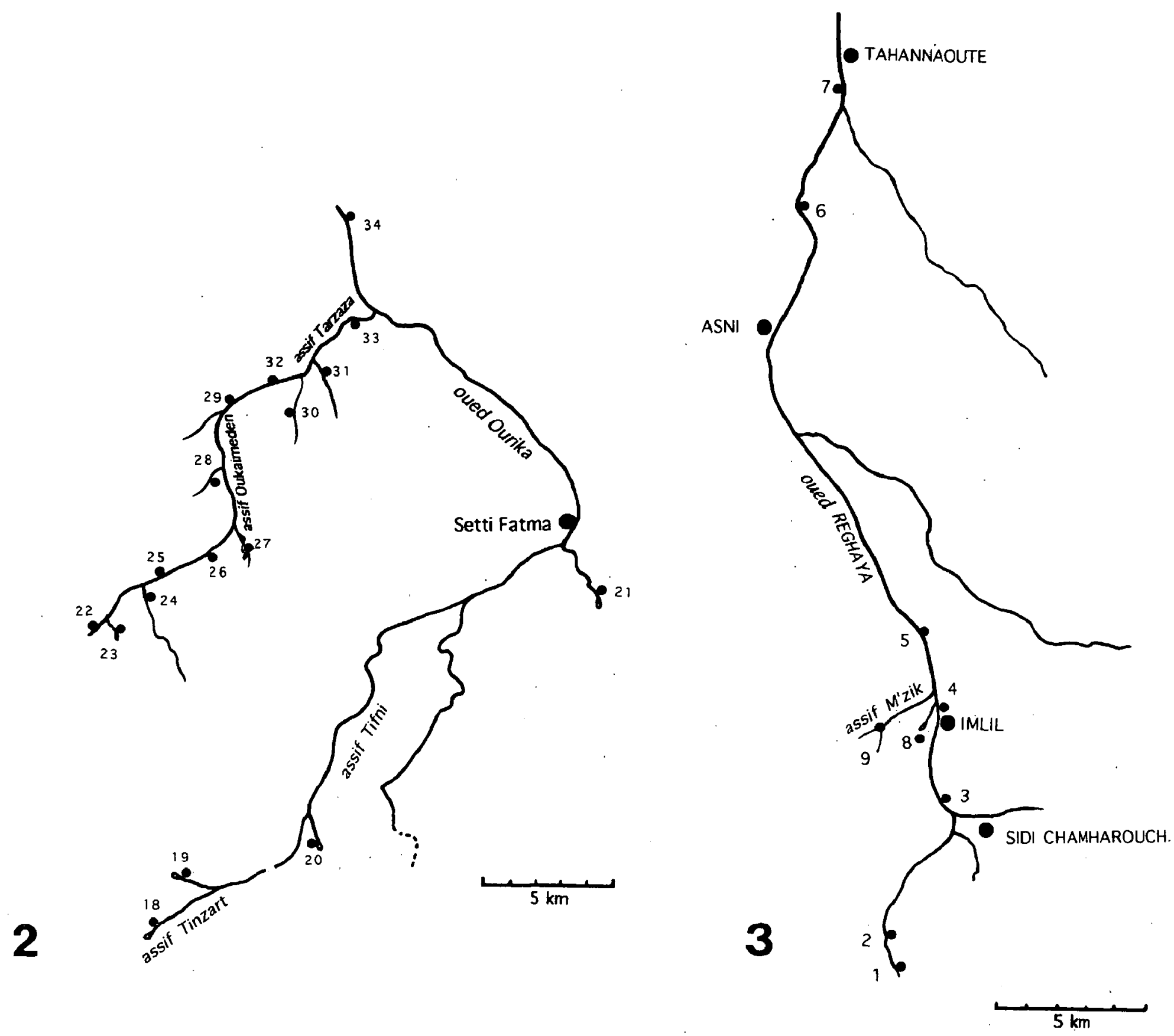

Figs. 2, 3. Cartes des réseaux hydrographiques de l'oued Ourika et de l'oued Reghaya et localisation des stations de prélèvements des Simulies. Figs 2, 3. Maps showing the Ourika wadi and the Reghaya wadi networks and the sites at which Simuliidae were collected.

- 20. ruisseau de Likemt (émissaire de source); $2670 \mathrm{~m}$.

(ces trois ruisseaux forment le secteur initial de l'Ourika)

-21. ruisseau de Tougroudadene (émissaire de source) débouchant dans l'oued Ourika en amont de Setti Fatma; $2660 \mathrm{~m}$.

-22. assif Oukaimeden, au nivau de la station de ski; $2600 \mathrm{~m}$.

-23. source hélocrène au niveau du cirque d'Oukaimeden; $2660 \mathrm{~m}$.

- 24. assif Tiferguine, affluent en rive droite de l'assif Oukaimeden; $2500 \mathrm{~m}$.
-25. assif Oukaimeden; $2450 \mathrm{~m}$.

- 26. ruisseau émissaire de source débouchant dans l'assif Oukaimeden; $1740 \mathrm{~m}$.

- 27. complexe rhéocrène formé par des émissaires de source débouchant dans l'assif Oukaimeden; $1730 \mathrm{~m}$.

-28. affluent temporaire en rive gauche de l'assif Oukaimeden; $1630 \mathrm{~m}$.

-29. affluent temporaire en rive gauche de l'assif Oukaimeden; $1360 \mathrm{~m}$.

-30. affluent temporaire en rive droite de l'assif $\mathrm{Ou}$ kaimeden; $1260 \mathrm{~m}$. 
- 31. affluent en rive droite de l'assif Oukaimeden; $1300 \mathrm{~m}$

- 32. assif Tarzaza (appellation de l'assif Oukaimeden dans sa partie inférieure), secteur temporaire; $1200 \mathrm{~m}$.

-33. assif Tarzaza; $1000 \mathrm{~m}$.

- 34. cours inférieur de l'oued Ourika, en amont de Souk Etnin; $850 \mathrm{~m}$.

- Réseau de l'oued Rdat

- 35. l'oued Rdat en amont de Taddert; $1850 \mathrm{~m}$.

- 36. affluent temporaire en rive gauche de l'oued Rdat; $1400 \mathrm{~m}$.

-37. l'oued Rdat; 1600 m.

- 38. l'oued Rdat; 1230 m.

- L'oued Tensift

- 39. l'oued Tensift entre 600 et $700 \mathrm{~m}$ d'altitude. (Trois stations ont été regroupées en une à cause de leur peuplement simulidien identique).

- 40. trois ruisseaux de khetarras (système d'irrigation drainant par gravité l'eau de la nappe phréatique de la plaine de Marrakech); 450 - $600 \mathrm{~m}$.

- Réseau de l'oued Tessaout

-41. l'oued Tessaout au niveau d'Ait Tamli; $1620 \mathrm{~m}$.

- 42. l'oued Lakdar en aval de la retenue de Sidi Driss; $1030 \mathrm{~m}$.

\subsection{Stations situées sur le versant sud.}

\section{- Réseau de l'oued Draa}

- 43. ruisseau émissaire de source formant le début de l'assif Imini; $2090 \mathrm{~m}$.

-44. l'assif Imini; $1560 \mathrm{~m}$.

-45. l'oued Ounila; $1820 \mathrm{~m}$.

- 46. ruisseau affluent en rive gauche de l'oued Ounila; $1820 \mathrm{~m}$.

-47. l'oued Ounila au niveau de Timlilt; $1600 \mathrm{~m}$.

- 48. l'oued Mellah au niveau d'Anghessa; $1400 \mathrm{~m}$.

- 49. l'oued Dadès en amont des gorges; 1630 m.

- 50. l'oued Dadès au niveau de Boumalne; $1530 \mathrm{~m}$.

- 51. l'oued Dadès au niveau de Sidi Flah; 1100 m.

- 52. l'oued M'goun; $1530 \mathrm{~m}$.

-53. l'oued M'goun an aval de Kelaa; $1370 \mathrm{~m}$.

- 54. ruisseau émissaire de source débouchant dans un affluent de l'oued Souss; $2350 \mathrm{~m}$.

Remarque : les stations prospectées ne subissent aucun impact de rejets polluants.

\section{Inventaire du peuplement simulidien du Haut Atlas}

\section{Liste des espèces}

Metacnephia blanci (Grenier \& Théodorides, 1953)

Prosimulium (Prosimulium) latimucro (Enderlein, 1925)

Simulium (Eusimulium) petricolum (Rivosecchi, 1953)

S. (E. ) velutinum (Santos Abreu, 1922)

S. (E.) mellah $\mathrm{n}$. sp.

Simulium (Nevermannia) ibleum (Rivosecchi, 1966)

$S .(N$.$) brevidens (Rubzov, 1956)$

$S$. (N.) costatum Friederichs, 1920

S. (N.) cryophilum (Rubzov, 1959)

$S$. (N.) latigonium (Rubzov, 1956)

$S .(N$.$) ruficorne Macquart, 1838$

S. (N.) toubkal Bouzidi \& Giudicelli, 1986

Simulium (Rubzovia) knidirii Giudicelli \& Thiéry, 1985

S. (R.) lamachi Doby \& David, 1960

Simulium (Obuchovia) marocanum Bouzidi \& Giudicelli, 1987

Simulium (Simulium) atlasicum Giudicelli \& Bouzidi, 1989

S. (S.) berberum Giudicelli \& Bouzidi 1989

S. (S.) intermedium Roubaud, 1906

S. (S.) ornatum Meigen, 1818

S. (S.) variegatum Meigen, 1818

S. (S.) xanthinum Edwards, 1933 (= S. gaudi Grenier \& Faure, 1957)

Simulium (Tetisimulium) bezzii (Corti, 1914)

Simulium (Wilhelmia) pseudequinum Séguy, 1921

S. (W.) sergenti Edwards, 1923

Sur les 29 espèces présentes au Maroc, 5 n'ont pas été récoltées dans le Haut Atlas ; ce sont Prosimulium (Prosimulium.) laamii Beaucournu-Saguez \& Bailly-Choumara, Prosimulium (P.) faurei Bernard, Grenier \& Bailly-Choumara, Simulium (Eusimulium) angustipes Edwards (= S.latizonum Rubzov), S.(Crosskeyellum) gracilipes Edwards, S.(Wilhelmia) quadrifila Grenier, Faure \& Laurent citées par BaillyChoumara \& Beaucournu-Saguez $(1978,1981)$. Il s'agit d'espèces cantonnées dans les régions septentrionnales du Maroc (massifs du Rif et du Moyen Atlas, Méséta côtière). Elles ont pour habitat des cours d'eau chauds de basse altitude et leur aire est limitée à la région occidentale du bassin méditerranéen (Afrique du Nord, sud de l'Espagne, Sicile).

Dans le matériel que nous avons récolté, six espèces se sont révélées être nouvelles pour la science. Parmi elles quatre sont, jüsqùà présent, endémiqües̃ de ce massif : $S$. knidirii, S. toubkal, S. atlasicum, S. berberum; elles repré- 
sentent les formes les plus sténothermes et les plus alticoles du peuplement simulidien de ce massif. Les deux autres espèces ( $S$. marocanum, $S$.(E.) mellah n. sp.) ont été trouvées aussi en Algérie (récoltes J. Gagneur et J. Giudicelli).

Deux espèces, $S$. petricolum Rivosecchi, $S$. brevidens Rubzov sont citées pour la première fois en Afrique du Nord.

\section{Description de Simulium mellah Giudi- celli \& Bouzidi n. sp.}

\section{Matériel examiné}

L'holotype $\sigma^{\top}$ et les paratypes sont extraits de pupes mûres. Holotype, 10 paratypes, 20 pupes, 15 larves, Maroc : oued Mellah, affluent de l'oued Ouarzazate (réseau de l'oued Draa), alt. $1400 \mathrm{~m}$, en amont du village d'Anghessa, 16.II.1986.

2 paratypes ơ, 10 pupes, 20 larves, Maroc : même localité, 8.VII 1985.

5 paratypes $\sigma^{\circ}, 15$ pupes, 20 larves, Algérie : oued Chiha el Ababda, $480 \mathrm{~m}$, affluent de l'oued Tafna, entre les localités de Sebra et Maghnia (wilaya de Tlemcen), 24.III.1988.

Tous les spécimens sont conservés dans l'alcool.

L'holotype, les paratypes et les paedotypes sont déposés dans la collection de J. Giudicelli (Marseille).

La nouvelle espèce appartient au sous-genre Eusimulium Roubaud, 1906 et au groupe de Eusimulium aureum Fries.

Dans la description, les caractères que partage la nouvelle espèce avec les espèces du groupe aureum ne sont pas mentionnés.

\section{Mâle}

Gonopodes (Fig. 4) très semblables à ceux de $S$. velutinum. Plaque ventrale (Fig. 5, 6) : bras très écartés, fortement sclérifiés et pigmentés, les deux bras forment un angle très ouvert; partie médiane étroite et pointue, en forme de triangle isocèle à bords rectilignes, pourvue latéralement et dorsalement de très nombreuses soies.

\section{Pupe}

Longueur du corps : 2,8-3,5 mm ; longueur du cocon: 4,6-5,2 mm. Cocon (Fig. 9, 10) en forme de chaussure, talon court, couleur jaune clair, tissage fin et serré, plus lâche près de l'ouverture, bord antérieur renforcé par un mince bourrelet. Appareil respiratoire (Fig. 9, 11) constitué de 4 filaments disposés en deux paires issues chacune d'un tronc basal très court ; filament supérieur présentant une forte courbure dans sa partie proximale. Soies et crochets abdominaux disposés comme dans le sous-genre Eusimulium.

\section{Larve mûre}

Longueur du corps : 5,2-6,4 mm. Couleur du corps gris noir. Capsule cépalique brun sombre ; fronto-clypeus (Fig. 12) présentant une grande tache centrale plus sombre sur un fond brun. Echancrure ventrale (Fig. 13) petite, bord antérieur légèrement arrondi. Submentum (Fig. 14) : bord antérieur sinueux, garni de 9 dents; la dent médiane et les deux dents latérales sont plus développées; bord latéraux présentant 5 ou 6 dents ; deux rangées latérales de 5 soies ( 3 grandes et 2 petites), la soie antérieure se terminant en pinceau. Eventail prémandibulaire : $40-49$ baguettes : disque postérieur formé de 62 à 65 rangées de 8 à 10 crochets.

\section{Affinités taxonomiques}

Simulium mellah se situe parmi les espèces du groupe aureum; l'espèce la plus proche est Simulium velutinum (Santos Abreu). Les caractères distinctifs les plus nets sont les suivants :

- au niveau des genitalia mâles, les gonopodes sont identiques à ceux de $S$. velutinum; par contre, la plaque ventrale de $S$. mellah, avec ses bras très écartés et sa partie médiane étroite, est bien caractéristique de l'espèce ;

- la forme en chaussure du cocon est unique dans le groupe aureum ;

- le filament supérieur de l'appareil respiratoire de la nymphe se courbe en arc de cercle dans sa partie basale ; chez $S$. velutinum la pliure fait un angle net ;

- la coloration sombre des larves et la pigmentation du fronto-clypeus différencient les larves de $S$. mellah de celles des autres espèces du groupe qui sont de coloration jaune clair.

\section{Notes écologiques}

Les deux cours d'eau où la nouvelle espèce a été récoltée sont caractérisés par des eaux très minéralisées et riches en sel (mellah signifie sel, en arabe). Les données hydrochimiques dont nous disposons pour la station de l'oued Mellah sont significatives :

conductivité : $9000 \mu \mathrm{S} . \mathrm{cm}^{-1}$;

chlorures : $2662 \mathrm{mg} . \mathrm{l}^{-1}$;

bicarbonates : $560 \mathrm{mg} . \mathrm{l}^{-1}$;

température de l'eau (le 8.VII.1985) : $28^{\circ} \mathrm{C}$.

Une espèce du même groupe aureum, $S$. (E.) salinum (Rubzov), a été trouvée en Sibérie, dans le même type d'habitat, avec des teneurs en sel de150-200mg. 1 $^{-1}$ (Rubzov 1956). 

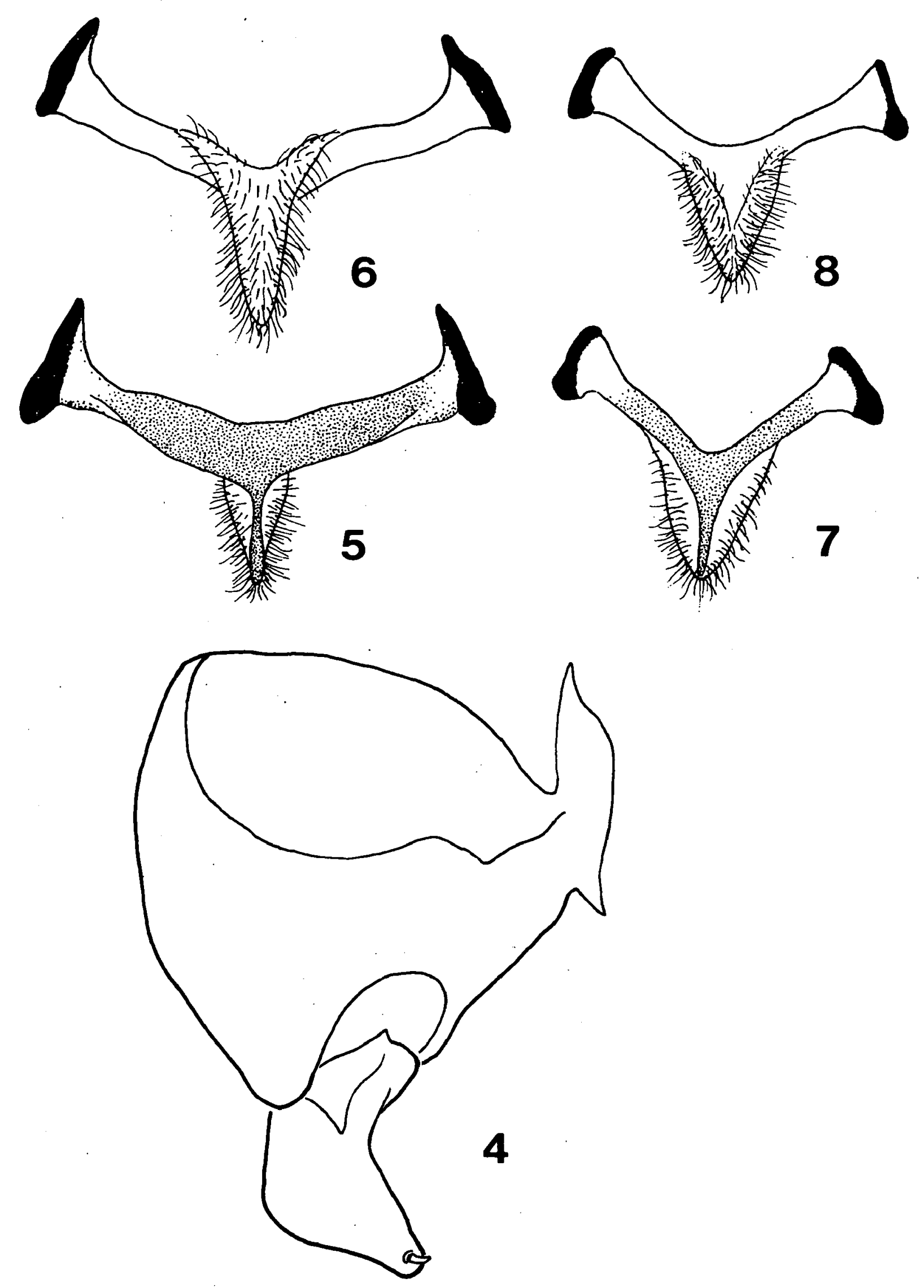

Figs. 4-8. Genitalia mâles de Simulium mellah n.sp. et $S$. velutinum (spécimen de l'oued Tensift, $600 \mathrm{~m}$ d'altitude). 4-6, $S$. mellah : 4, gonocoxite et gonostyle ; 5 et 6 , plaque ventrale, vue ventrale et vue dorsale. 7 et $8, S$. velutinum : plaque ventrale, vue ventrale et vue dorsale.

Figs. 4-8. Male genitalia of Simulium mellah sp.n. and S. velutinum (specimen from oued Tensift, $600 \mathrm{~m}$ a.s.1.). 4-6, S. mellah : coxite and style ; 5 and 6, ventral plate ventral view and dorsal view. 7 and $8, S$. velutinum : ventral plate, ventral view and dorsal view. 

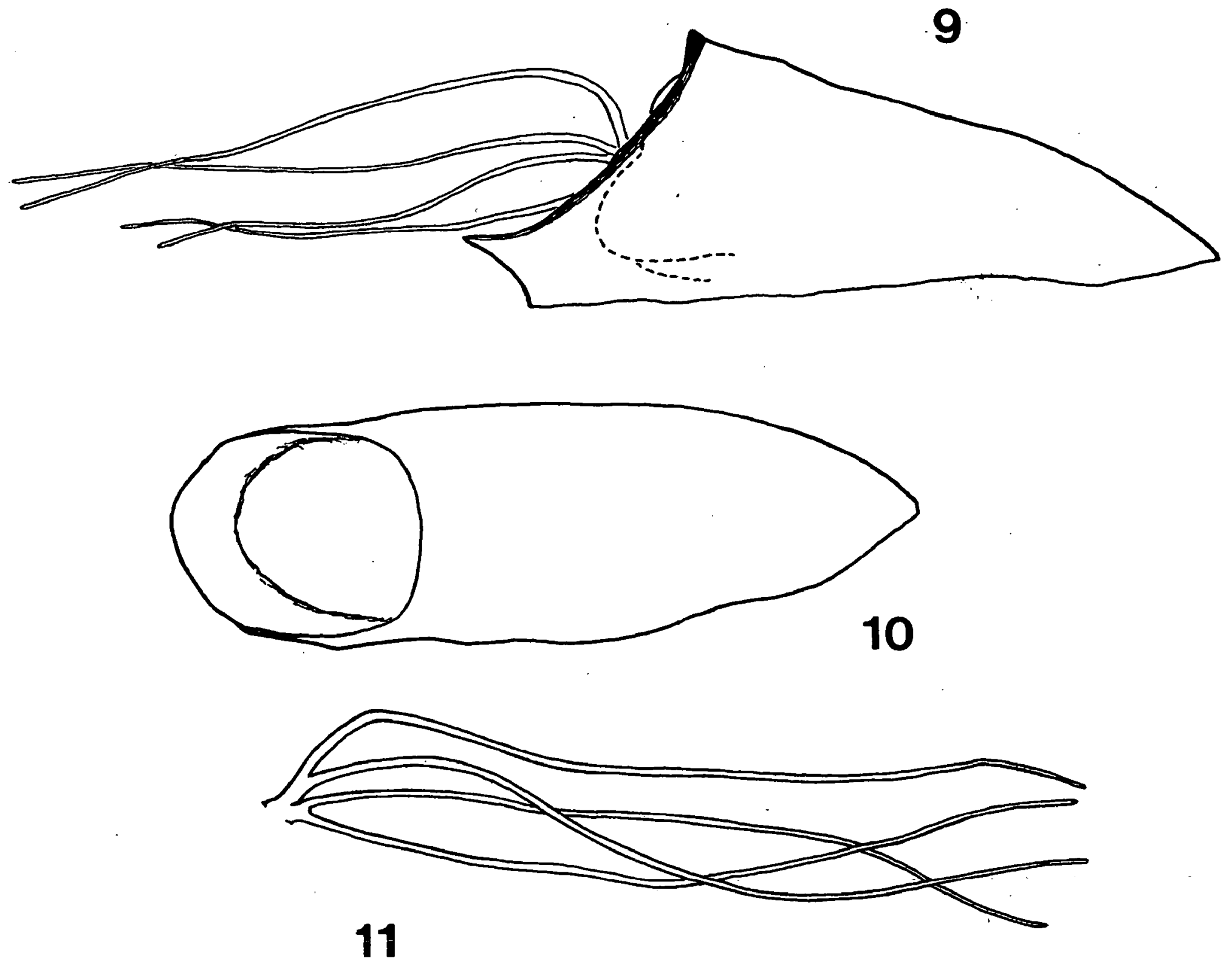

Figs. 9-11. Pupe de Simulium mellah n.sp.. 9 et $10:$ cocon, vue latérale et vue dorsale; 11 : filaments respiratoires.

Figs. 9-11. Pupa of Simulium mellah sp. n..9 and $10:$ cocoon, lateral view and dorsal view. 11 : pupal gills.

\section{Biogéographie du peuplement}

La faune des Simulies du Haut Atlas, comme celles des autres groupes d'invertébrés d'eau courante, est fondamentalement d'origine paléarctique. Boumaiza \& Clergue-Gazeau (1986), Gagneur \& Clergue-Gazeau (1988) ont fait la même remarque à propos des $\mathrm{Si}$ mulies de Tunisie et de l'ouest algérien.

Les espèces à large distribution européenne et s'étendant sur la région paléarctique occidentale sont les plus nombreuses.

Dans cette catégorie on trouve:

- Prosimulium latimucro, espèce à distribution boréo-alpine.

- Simulium petricolum. Son aire de distribution couvre l'Europe occidentale, les Balkans, l'Europe méditerranéenne (Italie, sud-est de la France, Corse, Pyrénées, Espagne). Sa présence dans le Haut Atlas (deux ruisselets de source) constitue la première citation de l'espèce en Afrique du nord.

- Simulium cryophilum. Espèce à vaste répartition paléarctique, bien représentée dans la région méditerranéenne et en Afrique du Nord.

- Simulium costatum. Cette simulie à vaste répartition européenne est considérée comme rare en Europe méridionale (Rivosecchi 1978). Absente en Tunisie, elle a été signalée par Grenier et al (1957) dans quelques stations du Moyen Atlas et du Rif, puis par Bailly-Choumara \& Beaucournu-Saguez (1981) dans trois stations du Haut Atlas; dans l'ouest algérien elle a été mentionnée dans dẹx stations (Gagneur \& Clergue-Gazeau 1988). 

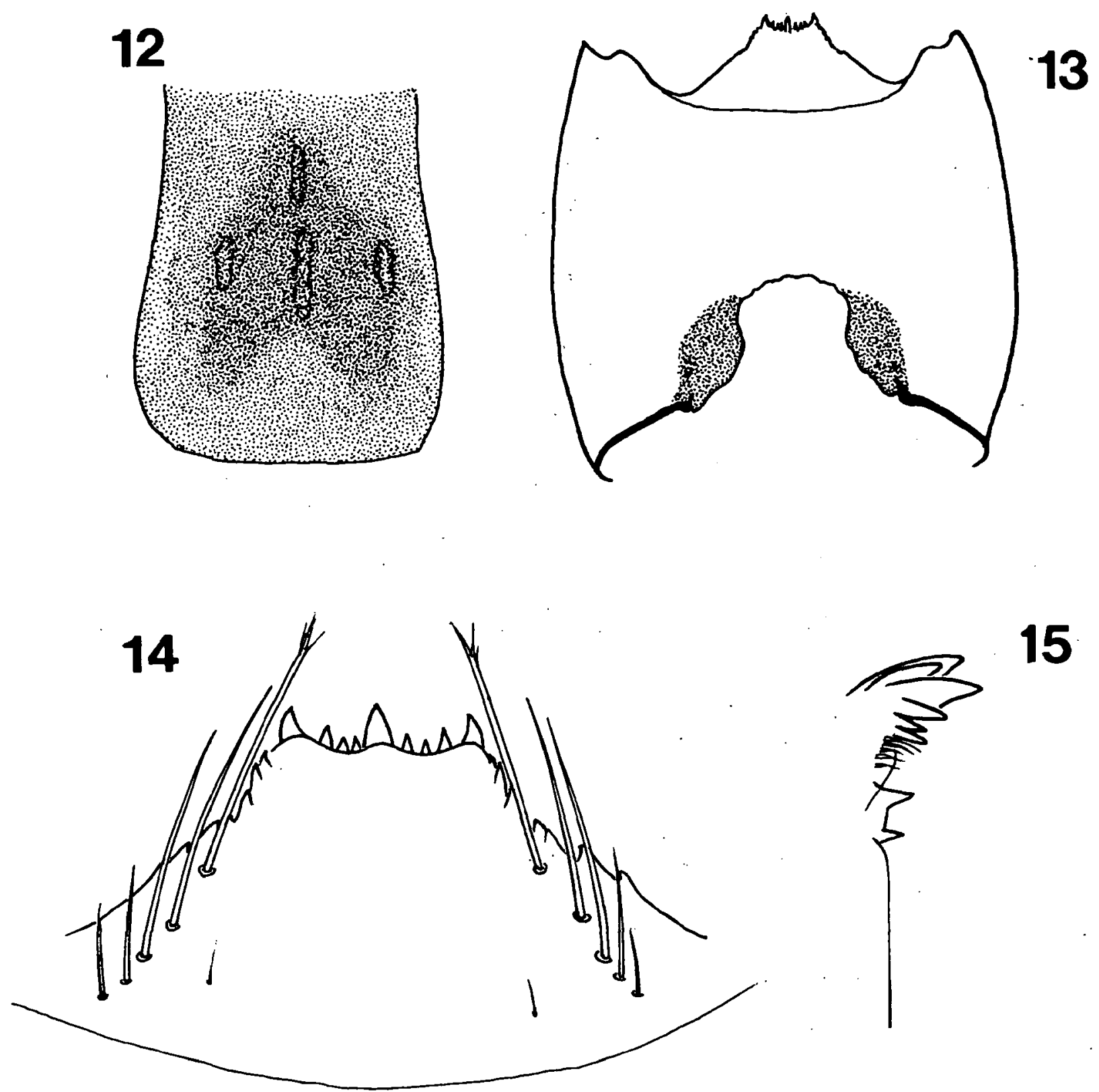

Figs. 12-15. Larve de Simulium mellah n.sp.. 12 : pigmentation du fronto-clypeus. 13 : capsule céphalique en vue ventrale montrant l'échancrure ventrale. 14 : submentum. 15 : extrémité de la mandibule.

Figs. 12-15. Larva of Simulium mellah sp. n.. $12:$ dorsal head pattern. $13:$ head ventral, showing postgenal cleft. $14:$ hypostomium. $15:$ tip of mandible.

- Simulium brevidens. Espèce connue surtout d'Europe orientale, sa présence dans trois de nos stations du Haut Atlas constitue la première citation pour l'Afrique du Nord.

- Simulium latigonium. Espèce euro-méditerranéenne, elle n'est connue, dans la partie méridionale de son aire, que de quelques rares stations en Italie (Rivosecchi 1978), dans les Pyrénées (Vinçon \& Clergue-Gazeau 1988) et en Espagne (Beaucournu-Saguez 1975). En Afrique du Nord elle a été trouvée pour la première fois en Algérie (Gagneur \& Clergue-Gazeau 1988). Dans nos récoltes elle ne figure que dans une seule station.
- Simulium variegatum. Elle est très commune dans les cours d'eau de montagne et de piémont de toute la zone paléarctique. Elle est assez rare en Afrique du Nord où elle n'a été trouvée que dans le haut Rif et sur le versant nord du Haut Atlas (Bailly-Choumara \& Beaucournu-Saguez 1978,1981).

- Simulium atlasicum, $S$. berberum. Ces deux endémiques du Haut Atlas font partie du groupe monticola représenté en Europe occidentale par quatre espèces sténothermes et montagnardes (S. argyreatum, S. monticola, S. maximum, S. sicana). Compte tenu de l'écologie des espèces du groupe, on comprend qu'elles se 
cantonnent, en Afrique du Nord, dans les plus hauts massifs : Haut Atlas marocain et Grande Kabylie (présence de Simulium gr. monticola selon Gagneur \& Clergue-Gazeau 1988).

Il est intéressant de noter que ces deux endémiques montrent les affinités les plus étroites avec Simulium niha Giudicelli \& Dia, endémique du Liban.

Neuf espèces confèrent au peuplement simulidien du Haut Atlas un caractère méditerranéen; ce sont des formes eurythermes ou thermophiles.

Parmi elles se trouvent trois espèces circumméditerranéennes :

- Simulium bezzii, espèce très commune sur tout le bassin méditerranéen, depuis le Maroc jusqu' au Liban.

- Simulium velutinum, dont l'aire s'étend sur l'Europe méditerranéenne et qui est très fréquente et abondante dans les cours d'eau d'Afrique du Nord.

- Simulium pseudequinum, qui occupe la frange méridionale de la zone paléarctique, depuis les Iles Canaries jusqu'à l'ouest de la Russie, en passant par l'Afrique du Nord, l'Espagne, le sud de la France, l'Italie, les Balkans, la Lybie, Israel, le Liban, le Pakis$\tan$.

Six espèces : Metacnephia blanci, S. ibleum, S. toubkal, S. xanthinum, S. marocanum, S. sergenti sont cantonnées dans la partie occidentale du bassin méditerranéen.

Metacnephia blanci est l'exemple type de cette distribution. Décrite sur des exemplaires provenant du Haut Atlas (Grenier \& Theodorides 1953), elle a été récoltée ultérieurement dans le Rif et dans divers cours d'eau du Haut Atlas (Bailly-Choumara \& BeaucournuSaguez 1978,1981). Elle se trouve aussi en Tunisie (Boumaiza \& Clergue-Gazeau 1986). Hors du Maghreb elle est rare puisqu'elle n'a été mentionnée que dans quelques stations en Espagne (Beaucournu-Saguez 1975), dans le midi méditerranéen français (Dorier 1963), en Sicile et dans les Balkans (Rivosecchi 1978).

S. toubkal est une endémique du Haut Atlas mais elle présente les plus grandes affinités taxonomiques avec $S$. rivosecchii, endémique de Sardaigne.

$S$. marocanum, décrite comme endémique marocaine (Bouzidi \& Giudicelli1987), a été trouvée depuis dans l'ouest algérien (Gagneur \& Clergue-Gazeau 1988). Elle constitue, avec $S$. galloprovincialis Giudicelli (sud-est de la France, Corse, Italie) et $S$. ibericum Crosskey \& Santos Gracio, endémique ibérique, une lignẹe ouest-méditerranéenne du sous-genre Obuchovia.
S. ibleum fait aussi partie de l'élément méditerranéen, son aire de distribution couvrant la partie méridionale du bassin (Sicile, Afrique du Nord, Lybie, Liban).

Trois espèces constituent l'élément le plus original de la faune des simulies du Haut Atlas car elles appartiennent ou se rattachent à des lignées éthiopiennes. Ce sont $S$. ruficorne, S. lamachi, S. knidirii.

L'aire de répartition de $S$. ruficorne couvre tout le continent africain, Madagascar, la Réunion, la plupart des régions méridionales du bassin méditerranéen: Maghreb, Egypte, Moyen Orient (Crosskey 1969). Le sud de la péninsule ibérique marque la limite septentrionnale de la distribution de l'espèce (BeaucournuSaguez 1972b). Elle a été trouvée aussi aux Iles Canaries (Crosskey 1988) et dans la péninsule arabique où elle monte jusqu'à $2500 \mathrm{~m}$ d'altitude (Crosskey \& Buttiker 1982). Au Maroc elle a été signalée d'abord dans les oasis sahariennes (Grenier et al 1957), puis dans le Rif et le Haut Atlas (Bailly-Choumara \& BeaucournuSaguez 1978, 1981).

Le sous-genre Rubzovia Petrova 1983 est remarquable par sa rareté (il n'est connu que de quelques stations isolées), par sa distribution géographique très fragmentée, par la nature particulière de l'habitat de ses stades aquatiques (ruisselets de source à très faible débit, coulant en une mince lame d'eau). Actuellement il réunit trois espèces.

L'espèce-type, S. lamachi, a été décrite d'une station dans les Pyrénées-Orientales à $700 \mathrm{~m}$ d'altitude, puis a été trouvée dans le sud du Massif Central à $690 \mathrm{~m}$, (Beaucournu-Saguez 1972a) et dans les Alpes françaises à $1000 \mathrm{~m}$ et à $1600 \mathrm{~m}$ (Bernard et al. 1974). En Afrique du Nord, elle n'est connue qu'au Maroc, dans une source du Rif à 1600 m (Giudicelli \& Dakki 1984) et une source dans la vallée de l'oued Reghaya (Haut Atlas) à $1750 \mathrm{~m}$. Récemment, Clergue-Gazeau \& Vinçon (1990) l'ont signalée dans une source du sud de l'Espagne.

S. knidirii n'est connue que du Haut Atlas. Décrite par Giudicelli \& Thiéry (1985) d'une petite source à $2660 \mathrm{~m}$ d'altitude, nous l'avons récoltée ultérieurement dans huit autres stations du crénal dans le Haut Atlas central entre 1400 et $3000 \mathrm{~m}$.

La troisième espèce, $S$. (R.) vantshi Petrova, a été découverte en Asie centrale, dans le massif du Pamir à $3300 \mathrm{~m}$ d'altitude.

Petrova (1983) a souligné les affinités taxonomiques de Rubzovia avec certaines espèces de Simulium d'Afrique tropicale: celles du sous-genre Pomeroyellum Rubzov 1962 et celles du groupe de S. griseicolle 
(sous-genre Byssodon Enderlein 1925). Elle pense que la distribution actuelle de Rubzovia, distribution paléarctique excluant la plus grande partie du massif alpin, les Carpathes et le Caucase, peut s'expliquer par la pénétration d'une forme ancestrale très ancienne depuis le continent africain jusqu'en Europe en passant par le Maroc (il s'agit de la lignée occidentale de lamachi-knidirii) et jusqu'en Asie à travers les montagnes de l'Asie Mineure (il s'agit de la lignée orientale de vantshi).

\section{Ecologie des espèces}

Cinq espèces forment le fond du peuplement simulidien du Haut Atlas car elles ont une fréquence supérieure à $40 \%$ et, de plus, elles constituent les populations larvaires les plus denses dans les secteurs de cours d'eau correspondant à leur optimum écologique. Ce sont $S$. bezzii, $S$. velutinum, $S$. intermedium, $S$. pseudequinum, $M$. blanci, espèces circum-méditerranéennes et ouest-méditerranéennes, à caractère nettement eurytherme.

$S$. bezzii et $S$. velutinum, parmi toutes les espèces récoltées, occupent la plus large zone altitudinale, depuis les stations les plus basses jusqu'à $2700 \mathrm{~m}$.

S. velutinum est, selon Jarry (1973), la simulie la plus adaptée aux contrastes du climat méditérranéen et, selon Beaucournu-Saguez (1975), la mieux représentée en Afrique du Nord. Dans les cours d'eau du Haut Atlas elle forme les populations d'invertébrés les plus abondantes au-dessous de $1600 \mathrm{~m}$.

$S$. bezzii, espèce typiquement méditerranéenne et hémisténotherme, est considérée en Europe comme caractéristique des cours d'eau rapides de la zone de piémont (Rivosecchi 1978). En Afrique du Nord, sa présence est liée aux grands massifs montagneux. En effet,elle est très répandue au Maroc, rare dans l'ouest algérien et absente en Tunisie. Dans les réseaux du Haut Atlas, comme dans ceux du Liban (Giudicelli \& Dia 1986), elle a un spectre écologique très large car elle constitue des populations abondantes dans presque tous les types d'eaux courantes, depuis le crénal jusqu'au potamal. Dans le Haut Atlas elle n'est exclue que des cours d'eau les plus froids (torrents au dessus de $2800 \mathrm{~m}$ d'altitude) et des cours d'eau à salinité élevée (oued Mellah, oued Tensift). Dans plus de la moitié des stations où figure l'espèce (33 stations sur 54) ses populations aquatiques ont une densité supérieure à 100 ind. $/ \mathrm{m}^{2}$; dans 7 stations situées sur de grandes rivières (cours inférieur de l'Ourika, de la Reghaya, du N'fis) la densité dépasse 1000 ind. $/ \mathrm{m}^{2}$.

$S$. intermedium est très fréquente au Maghreb. Elle est un élément dominant du peuplement simulidien dans les massifs marocains et dans l'ouest algérien, mais elle devient moins fréquente en Tunisie (Boumaiza \& Clergue-Gazeau 1986). Dans le Haut Atlas cette simulie est plus alticole et a un spectre écologique plus large (on la trouve dans tous les types de cours d'eau) qu'en Europe.

$S$. pseudequinum est une espèce plus thermophile que les précédentes; elle est mentionnée comme très commune dans les diférentes régions d'Afrique du Nord où elle prolifère dans les cours d'eau de plaine. En Italie et en Sicile sa limite altitudinale supérieure se situe vers $600 \mathrm{~m}$ (Rivosecchi 1978). Dans le Haut Atlas elle monte jusqu'à $1700 \mathrm{~m}$, mais elle est surtout abondante dans la partie inférieure des réseaux (le Tensift vers 600-700 m, l'Ourika au dessous de $900 \mathrm{~m}$, le $\mathrm{N}$ 'fis entre 600 et $1200 \mathrm{~m}$ ); elle réalise les plus fortes densités larvaires dans les grands cours d'eau du versant sud au dessous de $1500 \mathrm{~m}$.

L'aire de $M$. blanci couvre les régions les plus chaudes du bassin de la Méditerranée occidentale. En Afrique du Nord on la trouve régulièrement dans les réseaux du Haut Atlas, du Rif et du nord de la Tunisie; son absence des cours d'eau du Moyen Atlas et de l'ouest algérien doit être attribuée aux températures plus élevées des eaux. L'habitat des stades aquatiques de l'espèce, comme l'a indiqué Rivosecchi (1978), est bien caractéristique des rivières et grands torrents du Maroc avec un large lit pierreux soumis à des crues violentes. Dans le Haut Atlas l'espèce atteint $1850 \mathrm{~m}$ d'altitude et réalise son optimum écologique dans le cours moyen des grandes rivières entre 800 et $1500 \mathrm{~m}$. Ainsi, dans la Reghaya, la densité larvaire est de 5 à $30 / \mathrm{m}^{2}$ au dessus de $1500 \mathrm{~m}$, de 50 à $100 / \mathrm{m}^{2}$ vers 1200 $\mathrm{m}$, de $1000 / \mathrm{m}^{2}$ entre 900 et $1100 \mathrm{~m}$. La densité est faible dans les cours inférieurs: 5 à $10 / \mathrm{m}^{2}$ dans le N'fis à $650 \mathrm{~m}$. L'espèce est absente du Tensift où la température de l'eau dépasse $30^{\circ} \mathrm{C}$ en été. Elle est absente des sources et des petits ruisseaux et torrents, quelle que soit l'altitude.

Dans le classement hérarchique des espèces suivent quatre simulies dont la fréquence est comprise entre $21 \%$ et $33 \%$ (S. cryophilum, S. ornatum, S. variegatum, S. sergenti). Chacune occupe une gamme d'habitats et d'altitudes plus restreinte que celle des espèces précédentes.

$S$. cryophilum et $S$. ornatum ont une fréquence un peu supérieure à $30 \%$. La première est une forme montagnarde qui a été trouvée dans les émissaires de source entre 1750 et $2800 \mathrm{~m}$ et dans les petits cours d'eau à courant modéré du rhithral entre 1000 et $2600 \mathrm{~m}$. 
S. ornatum a une amplitude altitudinale plus large et se trouve dans des habitats plus variés : ruisseaux du crénal et du rhithral, cours inférieur des rivières de montagne. Elle présente une tendance plus alticole, plus sténotherme et plus rhithrophile que l'autre espèce du groupe ornatum, $S$. intermedium. Les deux espèces se trouvent généralement dans les mêmes stations. $S$. intermedium a été récoltée dans 24 stations, $S$. ornatum dans 17 stations; elles cohabitent dans 16 stations. S. intermedium vit seule dans les quatre stations inférieures du N'fis (entre 650 et $1600 \mathrm{~m}$ ) et dans deux stations de l'oued Ounila (entre 1600 et $1800 \mathrm{~m}$ ), secteurs caractérisés par des températures relativement élevées par rapport aux secteurs homologues des autres réseaux.

$S$. variegatum et $S$. sergenti ont respectivement une fréquence de $20 \%$ et $24 \%$. S. variegatum est commune en Europe dans les cours d'eau de basse et moyenne montagne entre 400 et $1000 \mathrm{~m}$, devenant plus rare à des altitudes supérieures. L'espèce est plus alticole dans le Haut Atlas où elle a pour habitat préférentiel les grandes rivières de montagne du versant nord entre 1000 et $2300 \mathrm{~m}$ (6 stations sur 11). Elle réalise ses effectifs les plus importants en juin dans la Réghaya entre 1550 et $2300 \mathrm{~m}$; en versant sud elle ne figure que dans un ruisseau de source à $2100 \mathrm{~m}$.

S. sergenti, simulie ibéro-magrébine, est largement répartie au Maroc, depuis le Rif jusqu'aux oueds des oasis présahariennes; les stades aquatiques vivent dans les cours d'eau eurythermes de la partie inférieure des réseaux. Dans le Haut Atlas nous l'avons récoltée dans 6 stations en versant nord entre 600 et $1250 \mathrm{~m}$ et dans 7 stations en versant sud entre 1050 et $1630 \mathrm{~m}$; elle a pour habitat exclusif les grands cours d'eau (Tensift, cours inférieur de la Réghaya, du N'fis, de l'Ourika, cours inférieur et moyen de l'oued Dadès, de l'oued Lakhdar, du Mgoun, de la Tessaout).

Neuf espèces ont une fréquence faible (de 6 à $15 \%$ ). Ce sont des espèces nettement sténoèces, inféodées à un type bien défini d'habitat; pour la plupart elles sont sténothermes.

S. toubkal, $S$. atlasicum, $S$. berberum habitent les torrents les plus froids, entre 1800 et 3200 d'altitude. S. toubkal a été trouvée dans 6 stations du versant nord entre 2400 et $3200 \mathrm{~m}$; peu abondante entre 2400 et $2800 \mathrm{~m}$, elle réalise son optimum écologique à des altitudes supérieures, dans les torrents où la température estivale est comprise entre 6 et $10^{\circ} \mathrm{C}$. Les deux espèces du groupe monticola, cantonnées dans la haute Réghaya, se succèdent en altitude $: S$. berberum est plus alticole (entre 2400 et $3200 \mathrm{~m}$ ) que $S$. atlasicum (entre 1800 et $2400 \mathrm{~m})$.
Trois espèces, $S$. costatum, $S$. ibleum, $S$. knidirii, sont strictement cantonnées dans les ruisseaux et ruisselets de source, à des altitudes comprises entre 1400 et $3000 \mathrm{~m}$. Des récoltes que nous avons effectuées en 1992 dans le massif du Siroua (extrémité occidentale de Haut Atlas) confirment ces constatations.

Le caractère crénobionte de $S$. costatum a été souvent mentionné : $\ll S$. costatum is an example par excellence of a species associated with rheocrenes » (Crosskey 1988). Les cinq stations où nous l'avons récoltée dans le Haut Atlas sont des émissaires de source. En Afrique du Nord elle n'a été trouvée, hors du Maroc, que dans deux ruisseaux de source de l'ouest de l'Algérie (Gagneur \& Clergue-Gazeau 1988).

Le crénal est l'habitat préférentiel de $S$. ibleum. Au Liban, Moubayed \& Clergue-Gazeau (1985) la mentionnent dans les émissaires de source vers $1000 \mathrm{~m}$ d'altitude et dans les eaux fraîches $\left(8,5\right.$ à $\left.13^{\circ} \mathrm{C}\right)$ des secteurs supérieurs du rhithral entre 1100 et $1360 \mathrm{~m}$. En Sicile elle vit dans les ruisseaux temporaires froids $\left(12^{\circ} \mathrm{C}\right.$ ) vers $600-700 \mathrm{~m}$ (Rivosecchi 1978). Dans le Haut Atlas nous l'avons trouvée dans la plupart des ruisseaux du crénal et dans un ruisseau temporaire à $1040 \mathrm{~m}$; la population la plus importante de l'espèce (250 à 400 ind. $/ \mathrm{m}^{2}$ ) vit dans une grande source rhéocrène à $1730 \mathrm{~m}$.

$S$. knidirii a été récoltée dans la plupart des sources prospectées. Découverte pour la première fois dans un ruisselet s'apparentant à un suintement (Giudicelli \& Thiery 1985), son biotope habituel est représenté par de petits émissaires de source à faible débit au dessus de $2000 \mathrm{~m}$.

Simulium marocanum et $S$. xanthinum sont des espèces relativement rares au Maroc et au Maghreb. Leur stades aquatiques semblent étroitement inféodés à un habitat particulier : les cascades des ruisseaux dont les eaux riches en bicarbonate de calcium forment des dépôts de tuf. Crosskey (1990 p. 202-203) a fait la même observation, dans le sud de l'Espagne, pour $S$. xanthinum et $S$. galloprovinciale Giudicelli 1963, espèce du même groupe que $S$. marocanum.

\section{Recherche des structures biocénotiques}

\subsection{Méthode d'analyse}

Une matrice des données faunistiques a été établie. Elle comporte 50 stations et 23 espèces (Tableau 2). Sur les 54 stations échantillonnées, 4 ont été exclues soit parce qu'elles hébergeaient une seule espèce (stations 1 et 23), soit parce qu'elles représentaient un milieu particulier (station 48 située sur un cours d'eau sa- 
Tableau 2. Matrice des données faunistiques (23 espèces x 50 stations)

Table 2. Faunistical data matrix (23 species $x 50$ stations).

\begin{tabular}{|c|c|c|c|c|c|c|c|c|c|c|c|c|c|c|c|c|c|c|c|c|c|c|c|}
\hline Stations & 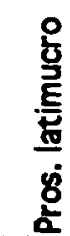 & 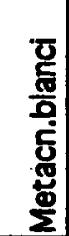 & $\begin{array}{l}\overline{\mathbf{t}} \\
\overline{\overline{0}} \\
\overline{5} \\
\dot{\text { si }}\end{array}$ & 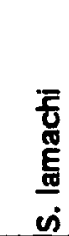 & 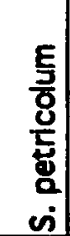 & $\begin{array}{c}\underline{\xi} \\
\mathbf{s} \\
\mathbf{5} \\
\mathbf{\$} \\
\dot{v}\end{array}$ & 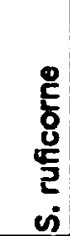 & $\begin{array}{l}\frac{\mathbf{p}}{\mathbf{0}} \\
\mathbf{0} \\
\mathbf{0} \\
\mathbf{5} \\
\dot{v}\end{array}$ & 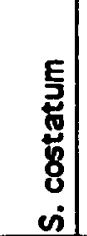 & 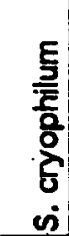 & 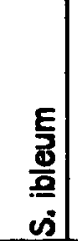 & 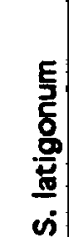 & $\begin{array}{l}\bar{s} \\
\bar{a} \\
\overrightarrow{3} \\
\dot{s}\end{array}$ & 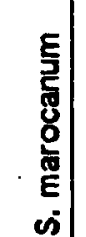 & 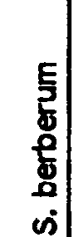 & $\begin{array}{c}\underline{E} \\
\frac{\vec{z}}{w} \\
\frac{m}{w} \\
\dot{w} \\
\dot{w}\end{array} \mid$ & 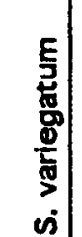 & $\begin{array}{c}E \\
\underline{E} \\
\underline{5} \\
\underline{5} \\
\dot{5} \\
v \\
v\end{array} \mid$ & 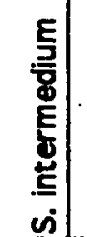 & 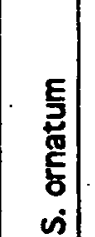 & {$\left[\begin{array}{c}\overline{\mathbf{V}} \\
\mathbf{D} \\
\mathbf{0} \\
v\end{array}\right]$} & 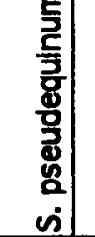 & 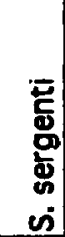 \\
\hline 2 & 1 & 1 & 1 & 1 & 1. & 1 & 1 & 1 & 1 & 1 & 1 & 1 & 6 & 1 & 5 & 1 & 1 & 1 & 1 & 1 & 1 & 1 & 1 \\
\hline 3 & 1 & 1 & 1 & 1 & 1 & 1 & 1 & 1 & 1 & 4 & 1 & 1 & 1 & 1 & 1 & 4 & 9 & 1 & 1 & 1 & 1 & 1 & 1 \\
\hline 4 & 1 & 2 & 1 & 1 & 1 & 3 & 1 & 1 & 1 & 3 & 1 & 1 & 1 & 1 & 1 & 3 & 7 & 1 & 1 & 2 & 8 & 4 & 1 \\
\hline 5 & 1 & 5 & 1 & 1 & 1 & 6 & 1 & 1 & 1 & 2 & 1 & 1 & 1 & $T$ & 1 & 1 & 5 & 1 & 1 & 1 & 7 & 5 & 1 \\
\hline 6 & 1 & 6 & 1 & 1 & 1 & 4 & 1 & 1 & 1 & $i$ & 1 & 1 & 1 & 1 & 1 & 1 & 1 & 1 & 1 & 1 & 4 & 3 & 4 \\
\hline 7 & 1 & 7 & 1 & 1 & 1 & 7 & 1 & $i$ & 1 & 1 & 1 & 1 & 1 & 1 & 7 & 1 & 1 & 1 & 1 & 1 & 7 & 7 & 5 \\
\hline 8 & 1 & 1 & 5 & 1 & 1 & 1 & 1 & 1 & 1 & 2 & 1 & 1 & 1 & 1 & 1 & 1 & 1 & 1 & 1 & 1 & 4 & 1 & 1 \\
\hline 9 & 1 & 1 & 3 & 2 & 3 & 2 & 1 & 1 & 3 & 3 & 2 & 1 & 1 & 1 & 1 & 1 & 7 & 1 & 2 & 2 & 4 & 1 & 1 \\
\hline 10 & 1 & 1 & 3 & 1 & 1 & 1 & 1 & 1 & 5 & 1 & 2 & 1 & 1 & 1 & 1 & 1 & 1 & 1 & 1 & 1 & 1 & 1 & 1 \\
\hline 11 & 1 & 1 & 1 & 1 & 1 & 1 & 1 & 1 & 1 & 1 & 1 & 1 & 4 & 1 & 6 & 1 & 1 & 1 & 1 & 1 & 1 & 1 & 1 \\
\hline 12 & 1 & 1 & 1 & 1 & 1 & 1 & 1 & 1 & 1 & 1 & 1 & 1 & 7 & 1 & 3 & 1 & 2 & 1 & 1 & 1 & 1 & 1 & 1 \\
\hline 13 & 1 & 1 & 1 & 1 & 1 & 3 & 1 & 1 & 1 & 4 & 1 & 1 & 2 & 1 & 3 & 1 & 1 & 1 & 1 & 1 & 1 & 1 & 1 \\
\hline 14 & 1 & 5 & 1 & 1 & 1 & 1 & 1 & 1 & 1 & 1 & 1 & 1 & 1 & 1 & 1 & 1 & 5 & 1 & 3 & 1 & 7 & 5 & 1 \\
\hline 15 & 1 & 6 & 1 & 1 & 1 & 4 & 1 & 1 & 1 & 1 & 1 & 1 & 1 & 1 & 1 & 1 & 5 & 1 & 3 & 1 & 9 & 6 & 1 \\
\hline 16 & 1 & 5 & 1 & 1 & 1 & 2 & 1 & 1 & 1 & 1 & 1 & 1 & 1 & 1 & 1 & 1 & 1 & 1 & 2 & 1 & 9 & 4 & 1 \\
\hline 17 & 1 & 3 & 1 & 1 & 1 & 2 & 3 & 1 & 1 & 1 & 1 & 1 & 1 & 1 & 1 & 1 & 1 & 1 & 2 & 1 & 4 & 4 & 4 \\
\hline 18 & 1 & 1 & 1 & 1 & 1 & 2 & 1 & 1 & 1 & 1 & 3 & 1 & 1 & 1 & 1 & 1 & 1 & 1 & 1 & 1 & 1 & 1 & 1 \\
\hline 19 & 1 & 1 & 2 & 1 & 1 & 1 & 1 & 1 & 1 & 2 & 1 & 1 & 1 & 1 & 1 & 1 & 1 & 1 & 1 & 1 & 1 & 1 & 1 \\
\hline 20 & 1 & 1 & 1 & 1 & 1 & 1 & 1 & 1 & 1 & 2 & 2 & 1 & 3 & 1 & 4 & 1 & 1 & 1 & 1 & 1 & 1 & 1 & 1 \\
\hline 21 & 1 & 1 & 4 & 1 & 1 & 1 & 1 & 1 & 1 & 1 & 1 & 1 & 3 & 1 & 5 & 1 & 1 & 1 & 1 & 1 & 1 & 1 & 1 \\
\hline 22 & 8 & 1 & 1 & 1 & 1 & 8 & 1 & 1 & 1 & 5 & 1 & 1 & 1 & 1 & 1 & 1 & 1 & 1 & 6 & 6 & 7 & 1 & 1 \\
\hline 24 & 1 & 1 & 1 & 1 & 1 & 9 & 1 & 1 & 1 & 1 & 1 & 1 & 1 & 1 & 1 & 1 & 1 & 1 & 9 & 9 & 8 & 1 & 1 \\
\hline 25 & 1 & 1 & 1 & 1 & 1 & 8 & 1 & 1 & 1 & 1 & 1 & 1 & 1 & 7 & 1 & 1 & 1 & 1 & 7 & 8 & 7 & 1 & 1 \\
\hline 26 & 2 & 1 & 2 & 1 & 1 & 1 & 1 & 1 & 1 & 6 & 5 & 1 & 1 & 1 & 1 & 1 & 1 & 1 & 2 & 2 & 1 & 1 & 1 \\
\hline 27 & 3 & 1 & 5 & 1 & 1 & 3 & 1 & 1 & 5 & 5 & 9 & 1 & 1 & 1 & 1 & 1 & 1 & 1 & 6 & 7 & 9 & 1 & 1 \\
\hline 28 & 1 & 1 & 1 & 1 & 1 & 1 & 1 & 3 & 1 & 6 & 1 & 1 & 1 & 1 & 1 & 1 & 1 & 1 & 5 & 3 & 5 & 1 & 1 \\
\hline 29 & 1 & 7 & 1 & 1 & 1 & 1 & 1 & 2 & 1 & 1 & 1 & 1 & 1 & 1 & 1 & 1 & 1 & 1 & 2 & 2 & 6 & 1 & 1 \\
\hline 30 & 2 & 2 & 1 & 1 & 1 & 2 & 1 & 1 & 1 & 1 & 1 & 1 & 1 & 1 & 1 & 1 & 5 & 2 & 4 & 3 & 3 & 1 & 1 \\
\hline 31 & 1 & 1 & 1 & 1 & 1 & 6 & 1 & 1 & 1 & 3 & 1 & 1 & 1 & 1 & 1 & 1 & 1 & 1 & 5 & 5 & 5 & 1 & 1 \\
\hline 32 & 2 & 3 & 1 & 1 & 1 & 2 & 1 & 1 & 1 & 4 & 5 & 7 & 1 & 1 & 1 & 1 & 3 & 2 & 2 & 2 & 7 & 1 & 1 \\
\hline 33 & 1 & 7 & 1 & 1 & 1 & 3 & 1 & 1 & 1 & 1 & 1 & 1 & 1 & 1 & 1 & 1 & 2 & 1 & 3 & 3 & 7 & 1 & 1 \\
\hline 34 & 1 & 5 & 1 & 1 & 1 & 7 & 1 & 1 & 1 & 1 & 1 & 1 & 1 & 1 & 1 & 1 & 1 & 1 & 7 & 7 & 9 & 8 & 9 \\
\hline 35 & 1 & 3 & 1 & 1 & 1 & 1 & 1 & 1 & 1 & 1 & 1 & 1 & 1 & 1 & 1 & 1 & 6 & 1 & 1 & 1 & 4 & 1 & 1 \\
\hline 36 & 1 & 1 & 1 & 1 & 1 & 2 & 1 & 1 & 1 & 3 & 1 & 1 & 1 & 6 & 1 & 1 & 1 & 1 & 1 & 1 & 1 & 5 & 1 \\
\hline 37 & 1 & 1 & 1 & 1 & 1 & 5 & 2 & 1 & 1 & 1 & 1 & 1 & 1 & 1 & 1 & 1 & 1 & 1 & 1 & 1 & 1 & 5 & 3 \\
\hline 38 & 1 & 6 & 1 & 1 & 1 & 3 & 1 & 1 & 1 & 1 & 1 & 1 & 1 & 1 & 1 & 1 & 1 & 1 & 1 & 1 & 6 & 1 & 1 \\
\hline 39 & 1 & 1 & 1 & 1 & 1 & 4 & 1 & 1 & 1 & 1 & 1 & 1 & 1 & 1 & 1 & 1 & 1 & 1 & 1 & 1 & 1 & 6 & 6 \\
\hline 41 & 1 & 4 & 1 & 1 & 1 & 4 & 1 & 1 & 1 & 1 & 1 & 1 & 1 & 1 & 1 & 1 & 1 & 1 & 4 & 1 & 7 & 2 & 2 \\
\hline 42 & 1 & 1 & 1 & 1 & 1 & 3 & 1 & 1 & 1 & 1 & 1 & 1 & 1 & 1 & 1 & 1 & 1 & 1 & 1 & 1 & 1 & 4 & 4 \\
\hline 43 & 4 & 4 & 3 & 1 & 2 & 1 & 1 & 2 & 1 & 5 & 2 & 3 & 1 & 1 & 1 & 3 & 2 & 1 & 5 & 1 & 6 & 1 & 1 \\
\hline 44 & 1 & 2 & 1 & 1 & 1 & 2 & 4 & 1 & 1 & 1 & 1 & 1 & 1 & 1 & 1 & 1 & 1 & 1 & 1 & 1 & 8 & 6 & 1 \\
\hline 45 & 1 & 4 & 1 & 1 & 1 & 1 & 1 & 1 & 2 & 1 & 1 & 1 & 1 & 1 & 1 & 1 & 1 & 1 & 5 & 2 & 5 & 1 & 1 \\
\hline 46 & 1 & 4 & 1 & 1 & 1 & 4 & 3 & 1 & 1 & 1 & 1 & 1 & 1 & 1 & 1 & 1 & 1 & 1 & 8 & 1 & 2 & 5 & 1 \\
\hline 47 & 1 & 2 & 1 & 1 & 1 & 6 & 1 & 1 & 1 & 1 & 1 & 1 & 1 & 1 & 1 & 1 & 1 & 1 & 8 & 2 & 2 & 6 & 1 \\
\hline 49 & 1 & 1 & 1 & 1 & 1 & 1 & 1 & 1 & 1 & 1 & 1 & 1 & 1 & 1 & 1 & 1 & 1 & 5 & 1 & 1 & 5 & 4 & 5 \\
\hline 50 & 1 & 1 & 1 & 1 & 1 & 2 & 4 & 1 & 1 & 1 & 1 & 1 & 1 & 1 & 1 & 1 & 1 & 1 & 1 & 1 & 4 & 9 & 5 \\
\hline 51 & 1 & 1 & 1 & 1 & 1 & 2 & 4 & 1 & 1 & 1 & 1 & 1 & 1 & 1 & 1 & 1 & 1 & 2 & 1 & 1 & 4 & 2 & 7 \\
\hline 52 & 1 & 3 & 1 & 1 & 1 & 6 & 2 & 1 & 1 & 1 & 1 & 1 & 1 & 1 & 1 & 1 & 1 & 1 & 1 & 1 & 7 & 3 & 5 \\
\hline 53 & 1 & 1 & 1 & 1 & 1 & 3 & 1 & 1 & 1 & 1 & 1 & 1 & 1 & 1 & 1 & 1 & 1 & 1 & 1 & 1 & 6 & 6 & 2 \\
\hline 54 & 1 & 1 & 1 & 1 & 1 & 1 & 1 & 1 & 5 & 1 & 1 & 1 & 1 & 1 & 1 & 1 & 1 & 1 & 3 & 3 & 1 & 1 & 1 \\
\hline
\end{tabular}


lé et très minéralisé, l'oued Mellah) ou artificialisé (station 48 correspondant aux ruisseaux de khetarras). Une espèce a été exclue, Simulium mellah, car elle a été recoltée seulement à la station 48 . Chaque couple espèce-station est affecté d'une classe d'abondance qui résulte du découpage des valeurs de densité correspondant au maximum d'abondance des espèces sur un cycle annuel ; le découpage des densités en classes d'abondance a été réalisé selon une progression logarithmique de raison 0,66 .

Les peuplements simulidiens des 50 stations retenues ont été traités par une analyse factorielle des correspondances (AFC) en utilisant le logiciel ADE (Doledec \& Chessel 1993).

Les trois premiers axes totalisent $49,42 \%$ de l'inertie totale du tableau des données.

\section{Taux d'inertie Inertie cumulée}

$\begin{array}{lll}\text { Axe } 1 & 19,60 \% & \\ \text { Axe } 2 & 17,33 \% & 36,93 \% \\ \text { Axe } 3 & 12,49 \% & 49,42 \%\end{array}$

Les axes 1 et 2 apparaissent complémentaires dans leur signification écologique. L'axe 1 oppose les espèces les plus microthermes et les stations les plus élevées en altitude et les plus froides, d'une part, aux espèces les plus euryèces et aux stations de piémont, d'autre part. L'axe 2 oppose des espèces hémisténothermes et des stations de moyenne montagne, d'une part, à des espèces thermophiles et aux stations les plus chaudes du cours inférieur des grands cours d'eau, d'autre part.

\subsection{Les groupements d'espèces et de stations}

Un programme de classification hiérarchique ascendante a mis en évidence deux ensembles majeurs au sein desquels s'individualisent des groupements d'espèces et de stations : trois groupements dans le premier ensemble, deux groupements dans le deuxième ensemble (Fig. 16, 17)

\section{Premier ensemble (Fig. $16 \mathrm{~B}$ ).}

Il occupe la moitié gauche du plan factoriel F1-F2 et réunit les espèces sténothermes et les milieux les plus froids. Les stations correspondant à cet ensemble se rapportent au crenal et au secteur supérieur du rhithral ; elles représentent tous les émissaires de source et presque tous les torrents de haute altitude.

Trois groupements d'espèces et de stations sont mis en évidence par l'analyse hiérarchique.

\section{Groupement 1}

Il isole les deux espèces les plus sténothermes et les plus alticoles, $S$. toubkal, $S$. berberum. Le groupement coenotique correspondant est constitué de cinq stations (Fig. 17) réparties sur quatre torrents froids et permaments de haute altitude (2660 et $3200 \mathrm{~m}$ ) :

- la station supérieure de la Réghaya (st. 2);

- les deux stations supérieures du réseau du N'fis (st. $11,12)$;

- les deux stations du cours supérieur de l'Ourika (st. 20, 21).

Stations de référence, composantes écologiques et faunistiques :

La station supérieure de la Réghaya $(3200 \mathrm{~m})$ et la station supérieure du réseau du N'Fis $(3000 \mathrm{~m})$ hébergent uniquement $S$. berberum et $S$. toubkal; ce sont des petits ruisseaux à faible débit $\left(201 . \mathrm{s}^{-1}\right)$, formant une succession de cascadelles sur un substrat de cailloux anguleux et de blocs, véhiculant des eaux très froides (4 à $10^{\circ} \mathrm{C}$ en été). L'autre station du N'fis $(2800 \mathrm{~m})$ contient, en plus des deux espèces précédentes, une petite population de $S$. variegatum, espèce commune dans les cours d'eau de montagne à des altitudes variées et dont la présence est ici en rapport avec un débit supérieur (210 à $\left.3801 . \mathrm{s}^{-1}\right)$.

Les deux stations du réseau supérieur de l'Ourika (st. 20 et 21 , alt. 2660 et $2670 \mathrm{~m}$ ) sont des ruisseaux émissaires de grandes sources rhéocrènes, avec des forts débits (100 à $\left.4001 . \mathrm{s}^{-1}\right)$, un écoulement rapide (60 à $\left.80 \mathrm{~cm} \cdot \mathrm{s}^{-1}\right)$, des eaux très froides $\left(4\right.$ à $6^{\circ} \mathrm{C}$ toute l'année) parce que alimentées par la fonte de névés permanents ; substrat constitué de blocs, de cailloux anguleux, de dalles; leur peuplement simulidien contient, en plus des deux espèces constituant le groupement faunistique, deux espèces crénobiontes, $S$. costatum et S. knidirii.

Le groupement 1 correspond donc au crénal et à l'épirhithral de haute altitude, représentés par des ruisseaux torrentiels froids (température de l'eau en été: 6 à $10^{\circ} \mathrm{C}$ ) alimentés par les eaux de fonte des névés ou par des sources rhéocrènes.

A ce groupement on peut rattacher la station 1 (non prise en compte dans cette AFC), située dans le bassin supérieur de la Reghaya, à $3800 \mathrm{~m}$. Elle représente un ruisseau très froid $\left(5^{\circ} \mathrm{C}\right.$ en août) issu d'un névé permanent qui héberge une seule espèce de simulie, Prosimulium latimucro, présente en été avec une importante population de larves et de pupes.

Sur le dendrogramme (Fig. $16 \mathrm{~A}$ ), les groupements 2 et 3 font partie d'un même sous-ensemble qui réunit 13 

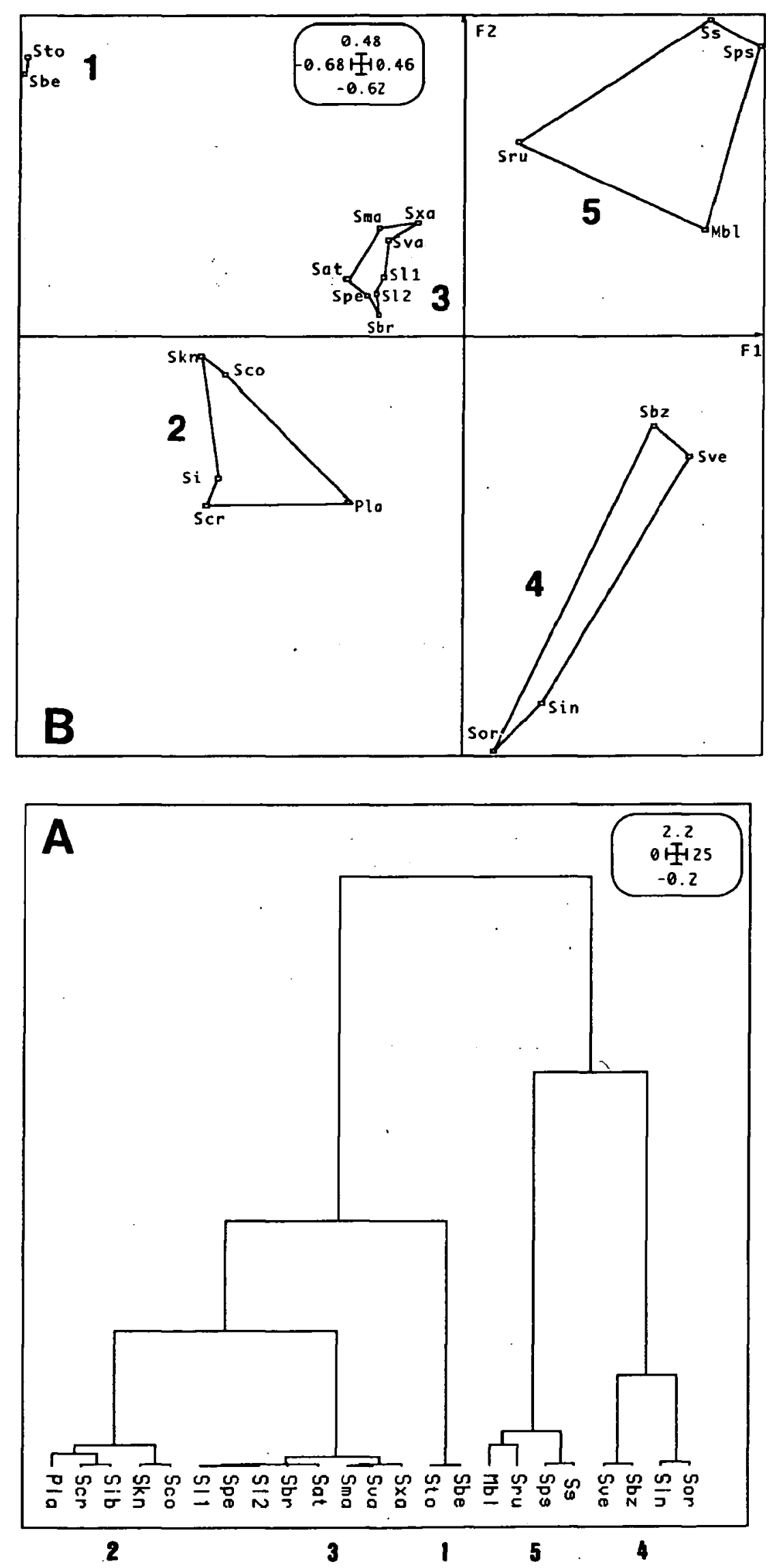

Fig. 16. Dendrogramme (A), distribution et groupement (B) des espèces dans le plan F1 x F2 de l'AFC.

Pla : Prosimulium latimucro, Scr : Simulium cryophilum, Sib : S. ibleum, Skn : S. knidirii, Sco : S. costatum, S11 : S. lamachi, Spe : S. petricolum, S12 : S. latigonium, Sbr: S. brevidens, Sat : S. atlasicum, Sma : S. marocanum, Sva : S. variegatum, Sxa : S. xanthinum, Sto : S. toubkal, Sbe : $S$. berberum, Mbl : Metacnephia blanci, Sru : S. ruficorne, Sps : S. pseudequinum, Ss : S. sergenti, Sve : S. velutinum, Sbz: $S$. bezzii, Sin: S. intermedium, Sor : S. ornatum.

Fig. 16. Dendrogram (A) and clustering (B) of species using FCA on the F1 x F2 factorial plane. 


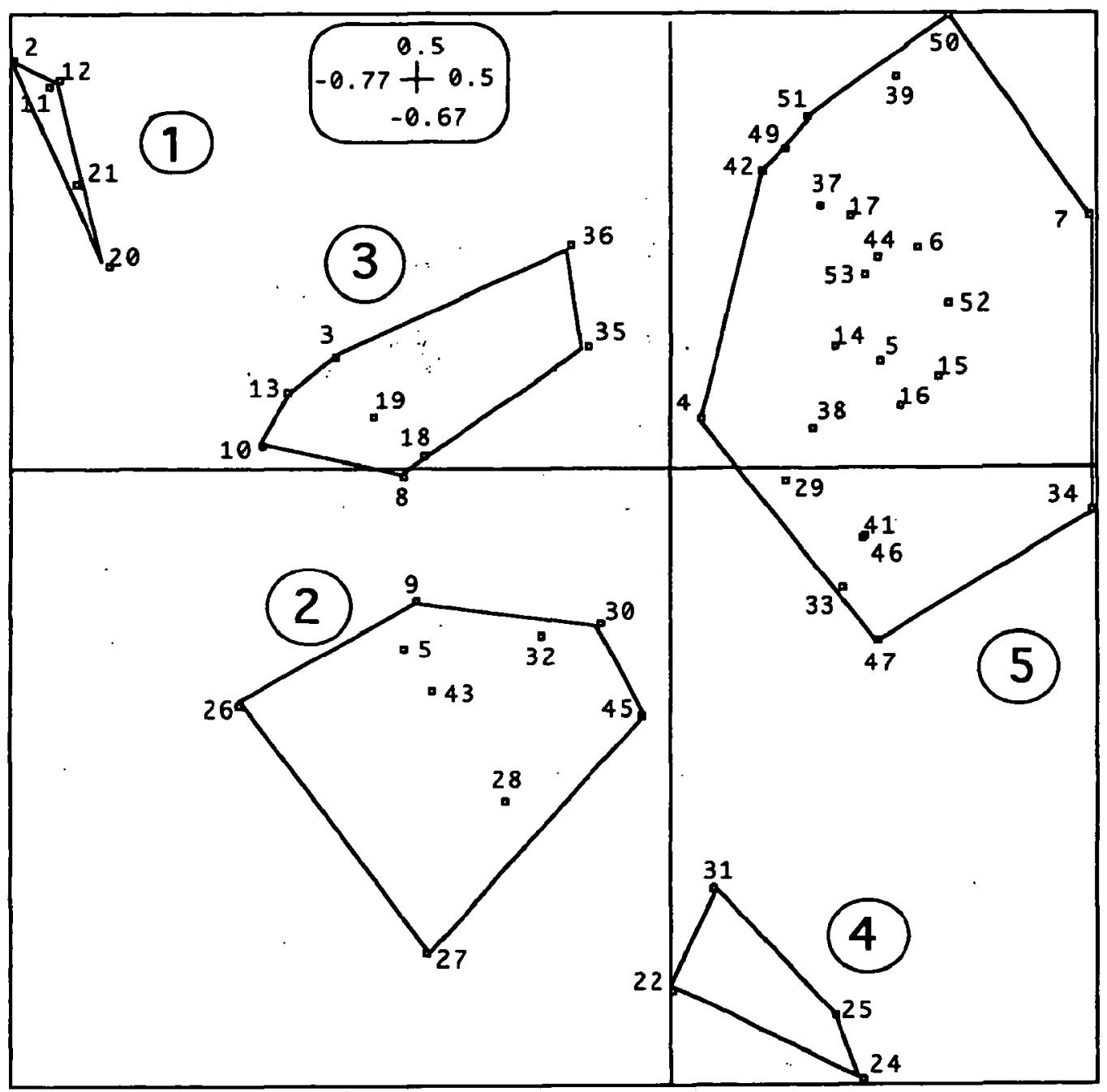

Fig. 17. Distribution et groupement des stations de prélèvements dans le plan F1 x F2 de l'AFC.

Fig. 17. Clustering of sampling sites using FCA faunistic data on the F1 x F2 factorial plane.

espèces sténothermes d'eau froide. A ce sous-ensemble correspondent 17 stations (Fig. 17) réparties dans la zone supérieure des réseaux hydrographiques à des altitudes comprises entre 1300 et $3000 \mathrm{~m}$. Neuf stations se situent sur des petits cours d'eau qui correspondent aux secteurs initiaux du rhithral (épi-métarhithral) et huit stations concernent des ruisseaux et ruisselets de source (hypocrénal).

\section{Groupement 2}

Il comprend cinq espèces : Simulium costatum, S.knidirii, S.cryophilum, S.ibleum, Prosimulium latimucro. Ce sont toutes des formes sténothermes d'eau froide; les deux premières sont crénobiontes strictes, les trois autres sont crénophiles.

\section{Les stations correspondantes sont :}

- 6 émissaires de source situés entre 1730 et $2350 \mathrm{~m}$, 3 en versant nord et 3 en versant sud ;
- 3 ruisseaux temporaires du réseau de l'Ourika, situés entre 1300 et $1630 \mathrm{~m}$.

Les stations se situent à des altitudes moins élevées (entre 1300 et $2090 \mathrm{~m}$ ) que celles du groupement 1 .

Au plan faunistique, ce groupement caractérise le crenon. Le peuplement simulidien des sources du Haut Atlas est défini par un groupe de quatre espèces : $\mathrm{Si}$ mulium costatum, S.knidirii, S.ibleum, S.cryophilum. Ces sources hébergent d'autres espèces sténothermes d'eau froide, réalisant ainsi les peuplements stationnels les plus riches - une dizaine d'espèces de simulies - que nous ayons relevés dans la région; c'est le cas du ruisseau de source près d'Aguelmous (station 43) qui héberge 11 espèces.

Les trois ruisseaux temporaires de ce groupement appartiennent à l'épirhithral; ils ont un peuplement simulidien identique ou très voisin de celui des émissaires de source car ils sont en eau pendant la saison froide et présentent alors les conditions écologiques favorables à une faune sténotherme. 
Stations de référence, composantes écologiques et faunistiques :

On retiendra trois stations.

1) Un émissaire de source du versant nord (st. 27) à $1730 \mathrm{~m}$ d'altitude, dans le réseau de l'Ourika. Largeur : 2,5 à $6 \mathrm{~m}$; débit : 80 à $1101 . \mathrm{s}^{-1}$; température de l'eau : 6 à $15^{\circ} \mathrm{C}$; substrat de blocs, cailloux et galets, important développement d'herbiers de macrophytes ( $R a$ nunculus aquatilis). La diversité de l'habitat aquatique détermine une richesse simulidienne élevée : neuf espèces Simulium ibleum, S.knidirii, S.bezzii, S.costatum, S.cryophilum, S.ornatum, S.intermedium, Prosimulium latimucro, S.velutinum (classées par densités décroissantes).

2) Un émissaire de source en versant sud (st. 43), à $2090 \mathrm{~m}$ d'altitude ; il s'agit du ruisseau de source qui forme le secteur initial de l'assif Imini. Largeur : 0,45 à $0,70 \mathrm{~m}$; débit : 19 à $211 \mathrm{~s}^{-1}$; température de l'eau : 8,1 à $12,2^{\circ} \mathrm{C}$; substrat de blocs et cailloux. Onze espèces : Simulium bezzii, S. cryophilum, Prosimulium latimucro, S. intermedium, $S$. knidirii, S. atlasicum, $S$. ibleum, $S$. brevidens, $S$. petricolum, $S$. variegatum, $S$. latigonium (classées par ordre de densités décroissantes).

3) Un ruisseau temporaire (st. $32,1300 \mathrm{~m}$ ), correspondant au cours inférieur de l'assif Oukaimeden, à sec de fin juin à novembre. Dix espèces : Simulium bezzii, S. ibleum, S. cryophilum, S. variegatum, Metacnephia blanci, Prosimulium latimucro, $S$. intermedium, $S$. ornatum, $S$. xanthinum, $S$. velutinum (classées par densités décroissantes). Cette communauté est composite : on y trouve une composante sténotherme avec $S$. cryophilum, $S$. ibleum et Prosimulium latimucro, une composante hémisténotherme avec $S$. variegatum, S. bezzii, S. ornatum, une composante caractéristique des cours d'eau temporaires avec Metacnephia blanci, S. ibleum, S. xanthinum.

\section{Groupement 3}

Il est constitué de huit espèces sténothermes (Simulium atlasicum, S. brevidens, S. lamachi, S.latigonium, $S$. petricolum) et hémisténothermes ( $S$. xanthinum, $S$. marocanum, $S$. variegatum). Ce sont toutes des espèces rares dans le Haut Atlas et dans les autres régions de leur aire géographique. Trois ont été trouvées dans une seule station (S. lamachi, S. latigonum, S. marocanum) et trois autres dans deux ou trois stations ( $S$. petricolum, S. brevidens, S. atlasicum).

Ce groupement est composite au plan écologique car il comprend :
- une espèce crénobionte (S. lamachi) et une espèce crénophile (S. brevidens) ;

- deux espèces dont la présence est en rapport avec des particularités hydrochimiques ( $S$. xanthinum, $S$. marocanum) ; de plus, elles vivent souvent dans des ruisseaux temporaires ;

- une espèce sténotherme d'eau froide (S. atlasicum);

- une espèce hémisténotherme caractéristique des rivières de montagne ( $S$. variegatum) ;

- une espèce inféodée aux petits ruisseaux steppiques ( $S$. petricolum).

Ce groupement d'espèces est déterminé par le peuplement de 8 stations de haute montagne (5 stations entre 2000 et $3000 \mathrm{~m}$; 3 stations entre 1600 et $1850 \mathrm{~m}$ ).

Quatre stations (st. 8, 10,18, 19) sont des émissaires de source de haute altitude (entre 1750 et $3000 \mathrm{~m}$ ) caractérisés par leurs dimensions réduites et un faible écoulement. Leur peuplement simulidien ne compte que deux ou trois espèces par station, mais il est caractérisé par la présence d'espèces crénobiontes $(S$. costatum, S. knidirii) et/ou crénophiles ( $S$. ibleum, S. cryophilum).

- Stations de référence, composantes écologiques et faunistiques :

Ruisselet émissaire de source à $3000 \mathrm{~m}$ (st. 10); débit très faible $\left(0,5\right.$ à $\left.11 \mathrm{~s}^{-1}\right)$; température de l'eau 13 à $15^{\circ} \mathrm{C}$ en été ; trois espèces : $S$. costatum, $S$. knidirii, $S$. ibleum (classées par ordre de densités décroissantes).

A ce type d'habitat on doit rattacher la station 23 (non prise en compte dans l'AFC), qui représente un ruisselet de source hélocrène et qui héberge une seule espèce de simulie, Simulium knidirii.

Deux stations (st. 3, 13) sont des ruisseaux de haute altitude ( 2300 et $2400 \mathrm{~m}$ ) situés dans la partie supérieure des réseaux de la Reghaya et du N'fis.

- Station de référence, composantes écologiques et faunistiques :

La Reghaya (st. 3). Largeur : 100-150 cm ; débit : 90-380 l s rature de l'eau 6 à $14^{\circ} \mathrm{C}$; substrat constitué de cailloux et de gros blocs plats ; trois espèces : Simulium variegatum, S. atlasicum, S. cryophilum (classées par ordre de densités décroissantes).

Deux stations (st. 35, 36) se situent, l'une sur le cours supérieur du Rdat à $1850 \mathrm{~m}$ d'altitude, l'autre sur un affluent du Rdat à $1400 \mathrm{~m}$. Cette station est intéressante car elle est caractéristique de l'habitat de Simulium marocanum, endémique maghrébine. 
- Station de référence, composantes écologiques et faunistiques :

station 36 : ruisseau temporaire, alternance de cascades et de vasques, température de l'eau : $11-17^{\circ} \mathrm{C}$; substrat formé par la roche mère au niveau des cascades (biotope de $S$. marocanum), de cailloux et de sable au niveau des vasques; quatre espèces : Simulium marocanum $S$. pseudequinum, $S$. cryophilum, $S$. velutinum (classées par ordre de densités décroissantes).

\section{DEUXIÈME ENSEMBLE}

Il est constitué de deux groupements d'espèces et de stations.

\section{Groupement 4}

Les points espèces et les points stations se situent dans le quadrant inférieur droit du plan F1-F2 (Figs 16 $B, 17)$. Le noyau faunistique est représenté par les quatre espèces les plus eurythermes et à large valence écologique : Simulium ornatum, $S$. intermedium, $S$. bezzii, S. velutinum.

Les quatre stations de ce groupement appartiennent au réseau de l'Ourika : trois stations $(22,24,25)$ sur le cours supérieur de l'assif Oukaimeden, entre 2450 et $2600 \mathrm{~m}$ d'altitude ; une station (31) sur un affluent temporaire de l'assif Tarzaza (dénomination de l'assif Oukaimeden dans son cours inférieur).

- Stations de référence, composantes écologiques et faunistiques :

Station 22. L'assif Oukaimeden à $2600 \mathrm{~m}$ d'altitude; largeur : 1,5 à $2 \mathrm{~m}$, température de l'eau entre 5 et $16^{\circ} \mathrm{C}$; substrat formé de cailloux et de blocs. Six espèces: Prosimulium latimucro, Simulium velutinum, $S$. bezzii, S. intermedium, S. ornatum, S. cryophilum . Prosimulium latimucro et $S$. cryophilum sont présentes en hiver et au début du printemps, les autres espèces leur succèdent au printemps et en été.

Station 25. L'assif Oukaimeden à $2450 \mathrm{~m}$ d'altitude. Largeur : 2 à $5 \mathrm{~m}$; température de l'eau entre 9 et $20^{\circ} \mathrm{C}$; substrat formé de cailloux et de blocs. Le peuplement réunit les quatre espèces constituant le groupement $4: S$. ornatum, $S$. velutinum, $S$. intermedium, $S$. bezzii (classées par ordre; de densités décroissantes).

\section{Groupement 5}

Les points-espèces et les points-stations se placent dans le quadrant supérieur droit du plan factoriel F1F2. Le noyau faunistique est constitué par quatre espèces thermophiles et potamophiles : Metacnephia blanci, Simulium pseudequinum, S. ruficorne, S. sergenti, associées aux stations des cours moyens et inférieurs des réseaux hydrographiques.

Les stations du versant nord se situent entre 600 et $1740 \mathrm{~m}$ d'altitude et pour la plupart entre 600 et 1200 m ; elles se situent sur les cours inférieur du N'fis (st $14,15,16,17$ ) de l'Ourika (st 33, 34), de la Reghaya (st 4, 5, 6, 7), du Tensift (st 39). Les stations du versant sud se situent entre 1030 et $1820 \mathrm{~m}$ et pour la plupart entre 1030 et $1630 \mathrm{~m}$; elles se rapportent à la partie terminale de l'oued Dadès (st 49,50,51), de l'oued Mgoun (st 52, 53), de la Tessaout (st 41, 42).

Le groupement 5 correspond au potamon.

Stations de référence, composantes écologiques et faunistiques :

On retient une station en versant nord et une en versant sud.

- La Reghaya à $890 \mathrm{~m}$ (st. 7). Cette station se situe à l'extrémité de la partie permanente du cours d'eau. Largeur : 1 à $10 \mathrm{~m}$; débit entre 0,3 et $21,5 \mathrm{~m}^{3} \cdot \mathrm{s}^{-1}$; température de l'eau entre 6 et $22^{\circ} \mathrm{C}$; conductivité : 190 à $650 \mu \mathrm{S} . \mathrm{cm}^{-1}$; substrat de cailloux, graviers et sable.

Cinq espèces: Simulium bezzii, Metacnephia blanci, $S$. pseudequinum, S. velutinum, S. sergenti.

- L'oued Dadès à $1530 \mathrm{~m}$ (st 50). Largeur : 3 à $10 \mathrm{~m}$; température de l'eau entre 13 et $28^{\circ} \mathrm{C}$; conductivité: 1000 à $1100 \mu \mathrm{S} . \mathrm{cm}^{-1}$; substrat de cailloux et sable grossier.

Cinq espèces : Simulium pseudequinum, S.sergenti, S.ruficorne, S.bezzii, S.velutinum.

Dans ce groupement on doit ajouter la station 40 (non prise en compte dans l'AFC) qui représente trois ruisseaux de khettaras, biotopes lotiques caractérisés par un débit faible et constant $\left(201 . \mathrm{s}^{-1}\right)$, un courant modéré (vitesse $<70 \mathrm{~cm} . \mathrm{s}^{-1}$ ), une température de $1^{\text {'eau }}$ constante dans le cycle annuel (autour de $20^{\circ} \mathrm{C}$ ). Le peuplement simulidien comprend trois espèces : Simulium intermedium, S. velutinum, S. ruficorne.

\section{Repartition des espèces dans les micro- habitats}

Au niveau de la station, la répartition des stades aquatiques de simulies dans un cours d'eau est régie par deux facteurs interdépendants, la vitesse du courant et la nature du susbtrat.

On trouve dans la littérature de nombreuses informations sur la présence des populations larvaires de certaines espèces en fonction de la vitesse du courant et de l'habitat (Burger 1986, Grenier 1949, Décamps et al. 1975, Phillipson 1956, Colbo 1979, Carlsson 1962 etc...). Dans la mesure où la vitesse du courant est en 
rapport direct avec le débit, la répartition des espèces est généralement analysée et interprétée en référence au type de cours d'eau défini par le stream order («order number is often mentionned in blackfly studies, because in any particular fauna it is usual for some species to inhabit entirely or mainly first-order streams and others to live in streams of higher order», Crosskey 1990 p. 184).

Au cours des recherches que nous avons menées sur les simulies du Haut Atlas nous avons étudié la répartition des espèces vivant dans une même station en fonction des microhabitats présents, ceux-ci étant définis par la combinaison du couple substrat-vitesse du courant. Deux stations ont été retenues sur le cours inférieur (hépipotamal) de deux rivières du versant nord, l'oued Ourika à $1000 \mathrm{~m}$ d'altitude, l'oued Zat à $1050 \mathrm{~m}$.

A la suite d'analyses granulométriques 4 types de substrats minéraux ont été reconnus (sables, graviers, galets, blocs) et deux substrats organiques (débris végétaux, herbiers aquatiques : renoncules, cressons, algues filamenteuses). Les vitesses du courant, mesurées à $5 \mathrm{~cm}$ au dessus du fond, ont été classées dans les 5 catégories de l'échelle de Berg (1948) : très lent, lent, modéré, rapide, très rapide. La combinaison des catégories de substrat et de vitesse de courant a conduit à identifier 18 microhabitats dans la station de l'Ourika et 16 microhabitats dans la station du Zat.

De décembre 1985 à décembre 1986, 3 prélèvements mensuels (surface de substrat échantillonnée à chaque prélèvement : $400 \mathrm{~cm}^{2}$ ) ont été réalisés dans chaque station et pour chaque microhabitat.

Ces prélèvements quantitatifs ont fourni 39 taxons de macroinvertébrés benthiques dont 6 espèces de simulies dans la station de l'Ourika, 51 taxons dont 7 espèces de simulies dans la station du Zat.

L'étude des relations existant entre les deux variables environnementales (courant, substrat) et la répartition des invertébrés benthiques a été conduite en utilisant une AFC. Deux matrices des données correspondant aux deux stations ont été établies : à chaque couple taxon-microhabitat est associée l'abondance maximale du taxon considéré ; les effectifs ont été ramenés à une surface de substrat de $1 \mathrm{~m}^{2}$. Les résultats de l'analyse montrent que, pour les deux stations, les taxons s'ordonnent sur l'axe 1 en fonction leur degré de rhéophilie (Tableaux 3 et 4).

Pour la station de l'Ourika, cet axe oppose les larves de Diptères Tabanidae (Tabanus), Stratiomyidae (Melanochelia), Chironomidae (Chironomini, Tanypodinae), qui vivent sur des substrats à granulométrie fine sous courant lent où elles trouvent les débris végétaux dont elles se nourrissent, aux taxons pétricoles et rhéobiontes que sont les larves et pupes de Diptères Blephariceridae (Liponeura) et de Simuliidae (Metacnephia blanci, Simulium bezzii), les Ephéméroptères Rhithrogena, Oligoneuriella. Le gradient de rhéophilie des taxons augmente depuis l'extrémité négative de l'axe jusquà l'extrémité positive. Sur le même axe on note aussi, dans le même sens, une opposition entre les microhabitats à granulométrie fine et ceux à granulométrie grossière.

Pour la station du Zat, le gradient de rhéophilie augmente depuis le côté positif de l'axe 1 jusqu'au côté négatif. On note une opposition entre, d'une part, les espèces lénitophiles : un Trichoptère Hydroptilidae (Oxyethira) dont les larves se nourrissent d'algues filamenteuses, un Coléoptère Haliplidae qui se nourrit aussi d'algues filamenteuses, des Diptères Ceratopogonidae (Forcipomyia) et Stratiomyidae (Melanochelia) dont les larves se nourrissent de fins débris organiques, un Gastéropode Planorbis et, d'autre part, des espèces très rhéophiles : Liponeura megalatlantica, Simulium bezzii, Metacnephia blanci, Limnius intermedius...

Sur un gradient de rhéophilie croissante, matérialisé par l'axe 1, les espèces de simulies des stations de l'Ourika et du Zat se remplacent dans le même ordre : Simulium velutinum, $S$. sergenti, $S$. intermedium, $S$. pseudequinum, $S$. ornatum, $S$. bezzii, Metacnephia blanci.

\section{Conclusions}

Les résultats de notre étude confirment et complètent des travaux réalisés sur les Simuliidae en région méditerranéenne.

Le peuplement simulidien du Haut Atlas s'est révélé d'un grand intérêt à deux titres :

- sa richesse taxonomique élevée comparée à celle des autres groupes d'invertébrés d'eaux courantes de ce massif ;

- le statut d'indicateur écologique qui s'attache à toutes les espèces.

Cette étude montre que le peuplement simulidien d'une région naturelle, ou même d'un réseau hydrographique, apporte une quantité d'informations comparable à celle des groupes d'invertébrés (Ephéméroptères, Plécoptères, Coléoptères, Trichoptères, autres Diptères) le plus souvent pris en compte dans l'écologie des eaux courantes.

Les données de la littérature sur l'écologie des Simulies sont maintenant suffisamment nombreuses et 
Tableau 3. Ordination des tảxons d'invertébrés (51 taxons d'invertébrés, incluses 7 espèces de Simulies) sur l'axe 1. Station de l'oued Zat.

Table 3. Ordination of the F1 scores (51 invertebrate taxa, included 7 blackfly species). Site of Zat wadi.

\begin{tabular}{|c|c|c|c|}
\hline $\begin{array}{l}\text { ESPECES } \\
\text { OU TAXONS }\end{array}$ & $\begin{array}{l}\text { GROUPES } \\
\text { TAXONOMIQUES }\end{array}$ & $\begin{array}{l}\text { CONTRIBUTIONS } \\
\text { RELATIVES (C. R.) }\end{array}$ & $\begin{array}{l}\text { COORDONNEES } \\
\text { FACTORIELES }\end{array}$ \\
\hline Oxyethira unidentata & Trichoptera & 57 & 2171 \\
\hline Haliplus lineaticollis & Coleoptera & 57 & 2171 \\
\hline Forcipomyia sp. & Dipt. Ceratopogonidae & 57 & 2171 \\
\hline Planorbis mitidjensis & Gastropoda & 51 & 1327 \\
\hline Melanochelia riparia & Dipt. Stratiomyidae & 54 & 1160 \\
\hline Psychoda sp. & Dipt. Psychodidae & 18 & 945 \\
\hline Lymnaea peregra & Gástropoda & 53 & 902 \\
\hline Tabanus sp. & Dipt. Tabanidae & 33 & 867 \\
\hline Hydroptila vectis & Trichoptera & 34 & 834 \\
\hline Micronecta meridionalis & Heteroptera & 33 & 673 \\
\hline Potamonectes clarki & Coleoptera & 22 & 590 \\
\hline Tubificidae & Oligochaeta & 38 & 552 \\
\hline Dasyhelea sp & Dipt. Ceratopogonidae & 27 & 529 \\
\hline Dryops striatellus & Coleoptera & 27 & 521 \\
\hline Simulium velutinum & Dipt. Simuliidae & 21 & 431 \\
\hline Tanypodinae & Dipt. Chironomidae & 35 & 395 \\
\hline Tanytarsini & Dipt. Chironomidae & 48 & 386 \\
\hline Mesophylax aspersus & Trichoptera & 5 & 363 \\
\hline Simullum sergenti & Dipt. Simuliidae & 2 & 276 \\
\hline Onychogomphus uncatus & Odonata & 7 & 205 \\
\hline Oulimnius rivularis & Coleoptera & 13 & 192 \\
\hline Dugesia gonocephala & Tricladida & 12 & 173 \\
\hline Wiedemannia sp. & Dipt. Empididae & 1 & 167 \\
\hline Baetis pavidus & Ephemeroptera & 1 & 72 \\
\hline Chironomini & Dipt. Chironomidae & 0 & 37 \\
\hline Hexatoma sp. & Dipt. Limoniidae & 0 & 29 \\
\hline Orthocladiinae & Dipt. Chironomidae & 0 & 7 \\
\hline Pisidium casertanum & Lamellibranchiata & 0 & 0 \\
\hline simulium intermedium & Dipt. Simuliidae & 0 & -44 \\
\hline Baetis navasi & Ephemeroptera & 0 & -67 \\
\hline Perla marginata & Plecoptera & 0 & -73 \\
\hline Simulium oseudequinum & Dipt. Simuliidae & 1 & $-11 i$ \\
\hline Caenis luctuosa & Ephemeroptera & 8 & -120 \\
\hline Setodes acutus & Trichoptera & 14 & -154 \\
\hline Perla bipunctata & Plecoptera & 1 & -181 \\
\hline Protonemura talboti & Plecoptera. & 1 & -186 \\
\hline Hydropsyche pellucidula & Trichoptera & 18 & -192 \\
\hline Ecdyonurus rothschildi & Ephemeroptera & 12 & -254 \\
\hline Baetis modani & Ephemeroptera & 53 & -277 \\
\hline Simulium ornatum & Dipt. Simuliidae & 15 & -321 \\
\hline Baetis maurus & Ephemeroptera & 79 & -493 \\
\hline Dicranota sp. & Dipt. Limoniidae & 4 & -523 \\
\hline Rhithrogena sp. & Ephemeroptera & 45 & -584 \\
\hline Agapetus dolichopterus & Trichoptera & 15 & -611 \\
\hline Metacnephia blanci & Dipt. Simuliidae & 45 & -672 \\
\hline Rhyacophila munda & Trichoptera & 8 & -708 \\
\hline Ancylus fluviatilis & Gastropoda & 13 & -712 \\
\hline Simullum bezii & Dipt. Simuliidae & 50. & -998 \\
\hline Limnius intermedius & Coleoptera & 24 & -1086 \\
\hline Liponeura megalatlantica & Dipt. Blephariceridae & 41 & -1393 \\
\hline Ephemerella ignita & Ephemeroptera & 21 & -1951 \\
\hline
\end{tabular}


Tableau 4. Ordination des taxons d'invertébrés (39 taxons d'invertébrés incluses 6 espèces de Simulies) sur l'axe 1. Station de l'óued Ourika.

Table 4. Ordination of the F1 scores (39 taxa of invertebrates, included 6 blackfly species). Site of Ourika wadi.

\begin{tabular}{|c|c|c|c|}
\hline $\begin{array}{l}\text { ESPECES } \\
\text { Ou TAXONS }\end{array}$ & $\begin{array}{l}\text { GROUPES } \\
\text { TAXONOMIQUES }\end{array}$ & $\begin{array}{l}\text { CONTRIBUTIONS } \\
\text { RELATIVES (C. R.) }\end{array}$ & $\begin{array}{l}\text { COORDONNEES } \\
\text { FACTORIEUES }\end{array}$ \\
\hline Liponeura megalatlantica & Dipt. Blephariceridae & 64 & 1275 \\
\hline Metacnephia blanci & Dipt. Simuliidae & 43 & 788 \\
\hline Simulium bezzii & Dipt. Simuliidae & 66 & 761 \\
\hline Ephemerella ignita & Ephemeroptera & 22 & 640 \\
\hline Simulium ornatum & Dipt. Simuliidae & 66 & 636 \\
\hline Rhithrogena sp. & Ephemeroptera & 8 & 464 \\
\hline Rhyacophila munda & Trichoptera & 4 & 441 \\
\hline Oligoneuriella skoura & Ephemeroptera & 9 & .384 \\
\hline Baetis maurus & Ephemeroptera & 56 & 364 \\
\hline Hydroptila vectis & Trichoptera & 1 & 189 \\
\hline Baetis rhodani & Ephemeroptera & 11 & 182 \\
\hline Perla bipunctata & Plecoptera & 1 & 178 \\
\hline Hydropsyche pellucidula & Trichoptera & 1 & -62 \\
\hline Simulium pseudequinum & Dipt. Simuliidae & 1 & -105 \\
\hline Orthocladiinae & Dipt. Chironomidae & 6 & -119 \\
\hline Baetis pavidus & Ephemeroptera & 1 & -157 \\
\hline Tanytarsini & Dipt. Chironomidae & 4 & -295 \\
\hline Hexatoma sp. & Dipt. Limoniidae & 2 & -360 \\
\hline Caenis luctuosa & Ephemeroptera & 31 & .447 \\
\hline Ecdyonurus rothschildi & Ephemeroptera & 15 & -449 \\
\hline Afroperlodes lecerfi & Plecoptera & 5 & -538 \\
\hline Oulimnius rivularis & Coleoptera & 12 & -576 \\
\hline Tubificidae & Oligochaeta & 13 & -580 \\
\hline Elmis maugetii & Coleoptera & 13 & -581 \\
\hline Hydropsyche fezana & Trichoptera & 13 & -587 \\
\hline Physa acuta & Gastropoda & 1 &.-647 \\
\hline Dicranota sp. & Dipt. Limoniidae & 6 & -653 \\
\hline Simulium velutinum & Dipt. Simuliidae & 9 & -671 \\
\hline Simulium sergenti & Dipt. Simuliidae & 18 & -685 \\
\hline$\overline{\text { Perla marginata }}$ & Plecoptera & 3 & -722 \\
\hline Wiedemannia sp. & Dipt. Empididae & 45 & -724 \\
\hline Hydropsyche maroccana & Trichoptera & 16 & -750 \\
\hline Dugesia gonocephala & Tricladida & 18 & -846 \\
\hline Forcipomyia sp. & Dipt. Ceratopogonida€ & 43 & -988 \\
\hline Tanypodinae & Dipt. Chironomidae & 63 & -1085 \\
\hline Potamonectes clarki & Coleoptera & 33 & -1243 \\
\hline Chironomini & Dipt. Chironomidae & 30 & $=1270$ \\
\hline Melanochelia riparia & Dipt. Stratiomyidae & 22 & -1270 \\
\hline Tabanus sp. & Dipt. Tabanidae & 38 & -1630 \\
\hline
\end{tabular}


concordantes pour qu'on puisse définir avec précision le statut écologique de chaque espèce.

Certaines espèces définissent un type de cours d'eau ou de zone écologique

- S. knidirii et $S$. lamachi sont inféodées au crénal à très faible débit et à écoulement lent ;

- S. costatum et $S$. ibleum ont pour habitat exclusif les émissaires de sources rhéocrènes ;

- S. petricolum est cantonnée dans les petits cours d'eau des zones steppiques d'altitude ;

- $S$. bezzii est typique des grandes rivières de montagne et de piémont ;

- M. blanci est adaptée aux conditions hydrologiques des cours d'eau méditerranéns, avec pour habitat des rivières de moyenne altitude subissant des épisodes hydrologiques très constratés avec des alternances de crues brutales et d'étiages sévères.

Certaines espèces sont indicatrices de conditions hydrochimiques : $S$. mellah est inféodée aux cours d'eau très riches en chlorures ; $S$. marocanum, $S$. xanthinum ont pour habitat les cascades des ruisseaux à eaux encroûtantes.

Certaines espèces sont indicatrices du régime thermique des cours d'eau :

- Prosimulium latimucro, S. toubkal, S. berberum sont de strictes sténothermes d'eau froide ;

- S. velutinum, S. pseudequinum, S. ruficorne, S. sergenti sont des formes thermophiles, abondantes dans les cours inférieurs.

Enfin cette étude montre que, si les stades aquatiques de Simuliidae sont des organismes fondamentalement adaptés aux eaux courantes, les espèces ont un rhéopréférendum défini. Il existe une série d'intermédiaires entre $S$. lamachi dont les stades immatures vivent dans des ruisselets qui s'apparentent à des suintements et des espèces comme $S$. marocanum caractéristique des cascades.

\section{Travaux cités}

Bailly-Choumara H., Bernard P., Grenier P., Le Roy-Muret M.C. \& Mouchet J. 1970. - Note faunistique sur les Simulies du nord de la Tunisie. Cah. O.R.S.T.O.M., sér. Entomol. médic. et Parasitol., 8 (4) : 377-382.

Bailly-Choumara H. \& Beaucournu-Saguez F. 1978. - Contribution à l'étude des Simulies (Diptera, Simuliidae) du Maroc. 1. Le Rif. Bull. Inst. Scient. Rabat, 3 : 121-144.

Bailly-Choumara H. \& Beaucournu-Saguez F. 1981. - Contribution à l'étude des Simulies (Diptera, Simuliidae) du Maroc. 2. Le Haut Atlas. Bull. Inst. Scient. Rabat, 5 : 39-57.

Beaucournu-Saguez F. 1972a. - Deuxième station de Simulium lamachei Doby et David 1960 : présence en Lozère, Massif Central. Ann. Parasitol., 47 : 169-173.
Beaucournu-Saguez F. 1972b. - Captures de Simulies dans la moitié sud du Portugal. Premières captures en Europe de Simulium sergenti Edwards 1923 et de Simulium ruficorne Macquart 1838. Anais da Escola Nacional de Sauda Publica e de Medicina Tropical, $6(1-4): 73-83$

Beaucournu-Saguez F. 1975. - Sur quelques Simulies (Diptera, Simuliidae) du nord-ouest de l'Espagne. Ann. Parasitol. Hum. et Comp., 50 (1) : 105-122.

Beaucournu-Saguez F. \& Braverman Y. 1987. - Greniera dobyi (Diptera, Simuliidae) espèce nouvelle du Golan (Israel). Ann. Parasitol. Hum. et Comp., 62 (1) : 77-91.

Berg K. 1948 - Biological studies on the river Susaa. Folia limnol. scand., $4: 318 \mathrm{p}$.

Bernard M.R., Grenier P. \& Bailly-Choumara H. 1972. — Description de Prosimulium (P.) faurei n. sp. (Diptera, Simuliidae). Cah. O.R.S.T.O.M., sér. Entomol. médic. et Parasitol., 10 (1) : 63-68.

Bernard M.R., Grenier P. \& Challier A. 1974. - Simulium lamachei et $S$. petricolum, espèces nouvelles pour les Alpes françaises. L'Entomologiste, $31: 19-20$.

Boumaiza M. \& Clergue-Gazeau M. 1986. - Le peuplement simulidien de la Tunisie. I. Inventaire, faunistique et biogéographie (Diptera, Simuliidae). Annls. Limnol., 22 (1) : 31-39.

Bouzidi A. 1989. - Recherches hydrobiologiques sur les cours d'eau des massifs du Haut Atlas (Maroc). Bio-écologie des macroinvertébrés et distribution spatiale des peuplements. Thèse d'Etat, Université d'Aix-Marseille III : 190 p.

Bouzidi A. \& Giudicelli J. 1986. - Contribution à l'étude faunsitique et écologique des Simulies (Diptera, Simuliidae) du Maroc I. Une nouvelle espèce du Haut Atlas : Simulium (Nevermannia) toubkal n. sp.. Annls Limnol., 22 (1) : 41-52.

Bouzidi A. \& Giudicelli J. 1987. - Contribution à l'étude faunistique et écologique des Simulies (Diptera, Simuliidae) du Maroc. П. Simulium (Obuchovia) marocanum n. sp. et les espèces méditerranéennes d'Obuchovia Rubzov. Annls Limnol., 23 (3) : 185 195.

Burger F. 1986. - Specialized habitat selection by black flies. in Black flies : ecology, population management and annotated world list. Kim K. C. \& M Meritt R.W. (eds.) Pennsylvania State University, University Park \& London: 528 p.

Carlsson G. 1962: - Studies on Scandinavian black flies (Fam. Simuliidae Latr.). Opusc. ent. Suppl. 21 : 1-279.

Chessel D. \& Doledec S. 1993. - A.D.E. version 3. 6. Hypercards stacks and programme library for the analysis of environnemental data. URA CNRS 1451, Université Lyon I. User's manual: 750 p.

Clergue-Gazeau M. \& Vinçon G. 1990. - Importance de la découverte de Simulium (Rubzovia) lamachi Doby et David en Péninsule Ibérique (Diptera, Simuliidae). Nouv. Revue Entomol. (N.S.), $7(3)$ : 303-306.

Clergue-Gazeau M., Lek S. \& Lek S. 1991 - Les Simulies d'Afrique du Nord. Nouvelles données sur la répartition de la faune du Maroç et biogéographie des espèces maghrébines (Diptera, Simuliidae). Revue Hydrobiol. trop., 24 (1) : 47-59.

Colbo M.H. 1979. - Distribution of winter-developing Simuliidae (Diptera) in eastern Newfoundland. Can. J. Zool., 57: 2143-2152.

Crosskey R.W. 1969. - A re-classification of the Simuliidae (Diptera) of Africa and its islands. Bull. Brit. Mus. (Nat. Hist.) (Entomology), Suppl. 14 : 1-195.

Crosskey R.W. 1988. - Taxonomy and geography of the blackflies of the Canary Islands (Diptera, Simuliidae). J. Nat. Hist., 22 : 321-355.

Crosskey R.W. 1990. — The natural history of blackflies. John Wiley \& Sons (ed.): 711 p.

Crosskey R.W. \& Buttiker W. 1982. - Insects of Saudi Arabia. Diptera : Fam. Simuliidaè. Fauna of Saudi Arabia, 4 : 398-446. 
Dakki M. 1979. - Recherches hydrobiologiques sur un cours d'eau du Moyen Atlas (Maroc). Thèse $3^{\text {ème }}$ cycle, Université d'AixMarseille III : $126 \mathrm{p}$.

Dorier A. 1962-1963. - Documents pour servir à la connaissance des Simuliidae du sud-est de la France, Trav. Labor. Hydrobiol. Univ. Grenoble 54-55 : 7-79.

Décamps H., Larrouy G. \& Trivellato D. 1975. - Approche hydrodynamique de la microdistribution d'invertébrés benthiques en eau courante. Annls Limnol., 11 : 79-100.

Edwards F.W. 1923. - On some algerian species of Simulium. Arch. Inst. Pasteur Algérie, 1 (4) : 647-653.

El Mezdi Z. \& Giudicelli J. 1986. - Etude d'un écosystème limnique peu connu : les khettaras de la région de Marrakech (Maroc). habitats et peuplements. Sciences de l'Eau, 6 (3) : 281-297.

Gagneur J. \& Clergue-Gazeau M. 1988. — Les Simulies d'Algérie (Diptera, Simuliidae). I. Premières données biogéographiques et écologiques sur les espèces de l'ouest algérien. Annls Limnol., 24 (3) : 275-284.

Giudicelli J. 1968. - Recherches sur le peuplement, l'écologie et la biogéographie d'un réseau hydrographique de la Corse centrale. Thèse es-Sciences Marseille, A.O. CNRS 2478 : 437 p.

Giudicelli J. \& Bouzidi A. 1989. - Contribution à l'étude faunistique et écologique des Simulies (Diptera, Simuliidae) du Maroc. III. Deux espèces nouvelles du Haut Atlas : Simulium (Simulium) atlasicum n. sp. et Simulium (Simulium) berberum n. sp.. Annls Limnol., 25 (2) : 145-158.

Giudicelli J. \& Dakki M. 1984. — Les sources du Moyen Atlas et du Rif (Maroc) : faunistique (description de deux espèces nouvelles de Trichoptères), écologie, intérêt biogéographique. Bijdragen tot de Dierkunde, 54 (1) : 83-100.

Giudicelli J. \& Dia A. 1986. - Contribution to the knowledge of the blackfly fauna (Diptera, Simuliidae) of Lebanon, with description of a new species and ecological comments. Aquat. Insects, 8 (3) : 123-139.

Giudicelli J. \& Thiery A. 1985. - About a peculiar type of rheocrene spring in the High Atlas (Morocco). Description of a simuliid characteristic of this habitat, Simulium (Crenosimulium n. g.) knidirii n. sp. (Diptera, Simuliidae). Bull. Zool. Mus. Univ. Amsterdam, 10, $15: 109-123$.
Grenier P. 1949. - Contribution à l'étude biologique des Simuliides France. Physiol. Compar. et Oecol., 1 : 165-330.

Grenier P. 1953. - Simuliidae de France et d'Afrique du Nord. Encyclopédie Entomologique 29, Lechevalier Paris (ed.) : 170 p.

Grenier P. \& Théodorides J. 1953. - Simulies (Diptera, Simuliidae) du Maroc. Arch. Inst. Pasteur Maroc, 4 (6) : 429-441.

Grenier P., Faure R. \& Laurent J. 1957. - Simulies (Diptera, Simu-

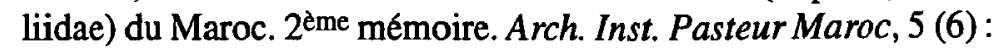
218-242.

Jarry D. 1973. - Contribution à l'étude des Simulies du Languedoc-Roussillon, I. E. latinum Rubzov et son écologie. Annls Parasitol. Hum. et Comp., 48 : 603-611.

Krno I. 1982. - Struktura a dynamika makrozoobenthosu riecky l'upcianky a jeji pritokov (Nizke Tatry) Biologcké Prace : $126 \mathrm{p}$.

Moubayed Z. \& Clergue-Gazeau M. 1985. - Les Simuliidae de trois rivières du Liban. Oronte, Litani et Beyrouth. Annls Limnol., 21 (1) : 83-88.

Parrot L. 1949. — Quelques notes sur les Simulidés d'Algérie. Arch. Inst. Pasteur Algérie, 27 : 273-276.

Petrova N.A. 1983. - A new genus and species of buffalognats (Diptera, Simuliidae) from West Pamir. Zoologichesky Zhurnal, $62,12: 1911-1915$. (en russe)

Phillipson J. 1956. - A study of factors determining the distribution of the larvae of the blackfly Simulium ornatum Mg. Bull. Entomol. Res., 47 : 227-238.

Rivosecchi L. 1978. - Simuliidae, Diptera Nematocera. Fauna d'Italia, vol. 13, Calderini (ed.), Bologna : 533 p.

Rubzov I.A. 1959-1964 - Simuliidae (Melusinidae), in Die Fliegen der Palearktischen Region, III (4), E. Lindner (Ed) : 689 p.

Vinçon G. 1987. - Comparaison de la faune benthique des vallées d'Aure et d'Ossau (Pyrénées) en vue de l'élaboration d'une méthodologie de surveillance des cours d'eau de montagne. Thèse de docteur ingénieur, Université Paul Sabatier, Toulouse : 381 p.

Vinçon G. \& Clergue-Gazeau 1988.- Etude hydrobiologique de la vallée d'Ossau (Pyrénées Atlantiques, France). III. Simuliidae (Diptera, Nematocera) : leur originalité biogéographique et écologique. Annls Limnol., 24 (1) : 67-81.

Zwick H. 1978. - Simuliidae (Diptera) in Limnofauna Europaea 2nd edit., Illies J. (ed.) : 396-403. 